\title{
A modern assessment of Ordovician chitinozoans from the Shelve and Caradoc areas, Shropshire, and their significance for correlation
}

\author{
THIJS R. A. VANDENBROUCKE* †, ANTONIO ANCILLETTA*, RICHARD A. FORTEY§ \\ \&JACQUES VERNIERS*
}

*Research Unit Palaeontology, Ghent University, Krijgslaan 281/S 8, 9000 Ghent, Belgium

$\S$ The Natural History Museum, Cromwell Road, London SW7 5BD, UK

(Received 20 April 2007; accepted 9 November 2007; First published online 28 November 2008)

\begin{abstract}
New chitinozoan data are presented from the classical section along the Onny River in the type Caradoc area, and from the deeper-water sections in the Shelve area, including the former British candidate GSSP for the base of the Upper Ordovician Series. The rich and well-preserved chitinozoan fauna of the Onny River has been a standard for 40 years, but new data revise some of the identifications. The assemblages are now attributed to biozones that are more readily applicable for international correlation. The main part of the section can be interpreted as belonging to the originally Baltoscandian Spinachitina cervicornis Biozone, although this is uncertain in the lower part. Within this biozone, the Fungochitina actonica Subzone has been defined. The Onny Formation at the top of the section is equated with the Acanthochitina latebrosa-Ancyrochitina onniensis Biozone; contrary to earlier reports, Acanthochitina barbata is absent. The Lower Wood Brook and Spy Wood Brook section from the Shelve Inlier yielded a great number of moderately to well-preserved chitinozoans, but a low-diversity assemblage. Their ranges have been neatly positioned against the well-known graptolite stratigraphy in the area. A local Eisenackitina rhenana Biozone? has been recognized, allowing us to suggest some international correlations.
\end{abstract}

Keywords: chitinozoans, biostratigraphy, Caradoc, Welsh Borderland, type section.

\section{Introduction and geological setting}

\section{1.a. Shropshire and the Welsh Borders}

Ordovician successions on either side of the Pontesford-Linley Fault are significantly different. West of this structure, the main, thick Shelve area sequence (Fig. 1a) displays a more or less complete record of Tremadoc, Arenig, Llanvirn and Caradoc rocks. In contrast, east of the Pontesford-Linley Fault, the thin, shallow marine Caradoc successions at Pontesford Hill and in the Caradoc type area (Fig. 1a) rest unconformably on rocks of Precambrian to Tremadocian age (Whittard, 1979; Fortey et al. 2000). An interpretation explaining the discrepancies states that the Pontesford-Linley Fault was active during preCaradoc times, forming the southeastern margin of the Welsh basin. The basin in which the deposition of the Shelve sediments took place lay west of the shoreline, while the area east of it was emergent as part of the Midland Platform. The early Caradoc sea-level rise, the gracilis transgression, initiated deposition also in the area east of the lineament, giving rise to the deposits of the type Caradoc area (Whittard, 1979; Woodcock, 1984). Alternatively, substantial displacement along the Pontesford-Linley Fault system, during Caradoc

$\nmid$ Postdoctoral Fellow of the Research Foundation-Flanders (FWOVlaanderen).

$\ddagger$ Author for correspondence: thijs.vandenbroucke@ugent.be times or later, might have caused/enhanced the shelfbasin contrast, juxtaposing terranes that were widely separated during most of the Ordovician (Woodcock, 1984; Woodcock \& Gibbons, 1988, p. 917).

\section{1.b. The Shelve Inlier}

The outcrop area of the Ordovician rocks of the Shelve Inlier covers a surface of some $111 \mathrm{~km}^{2}$ in Shropshire and the Welsh county of Powys (Fig. 1b). The area is more or less centred around the village of Shelve, and geologically bounded by the Pontesford-Linley Fault in the east, and by overstepping lower Silurian rocks at the southern and northern edges (Whittard, 1979). The westerly dipping succession consists of marine shelf sediments, intercalated with volcanic deposits. The continuously fossiliferous successions have drawn the attention of geologists ever since Murchison's time, and an overview of previous research on the area has been given in several papers by Whittard (1931, 1952, 1979). The 1979 paper provides a detailed geological map of the area, as well as a full description of the Ordovician rocks, with the exception of the Tremadoc Shineton Shale Formation and the Habberley Formation.

Following Whittard (1979), three important taxonomic studies have been carried out on material from the Shelve area. The studies by Strachan (1986) and by Hughes (1989) deal exclusively with graptolites. A third major contribution by R. M. Bettley (unpub. Ph.D. 


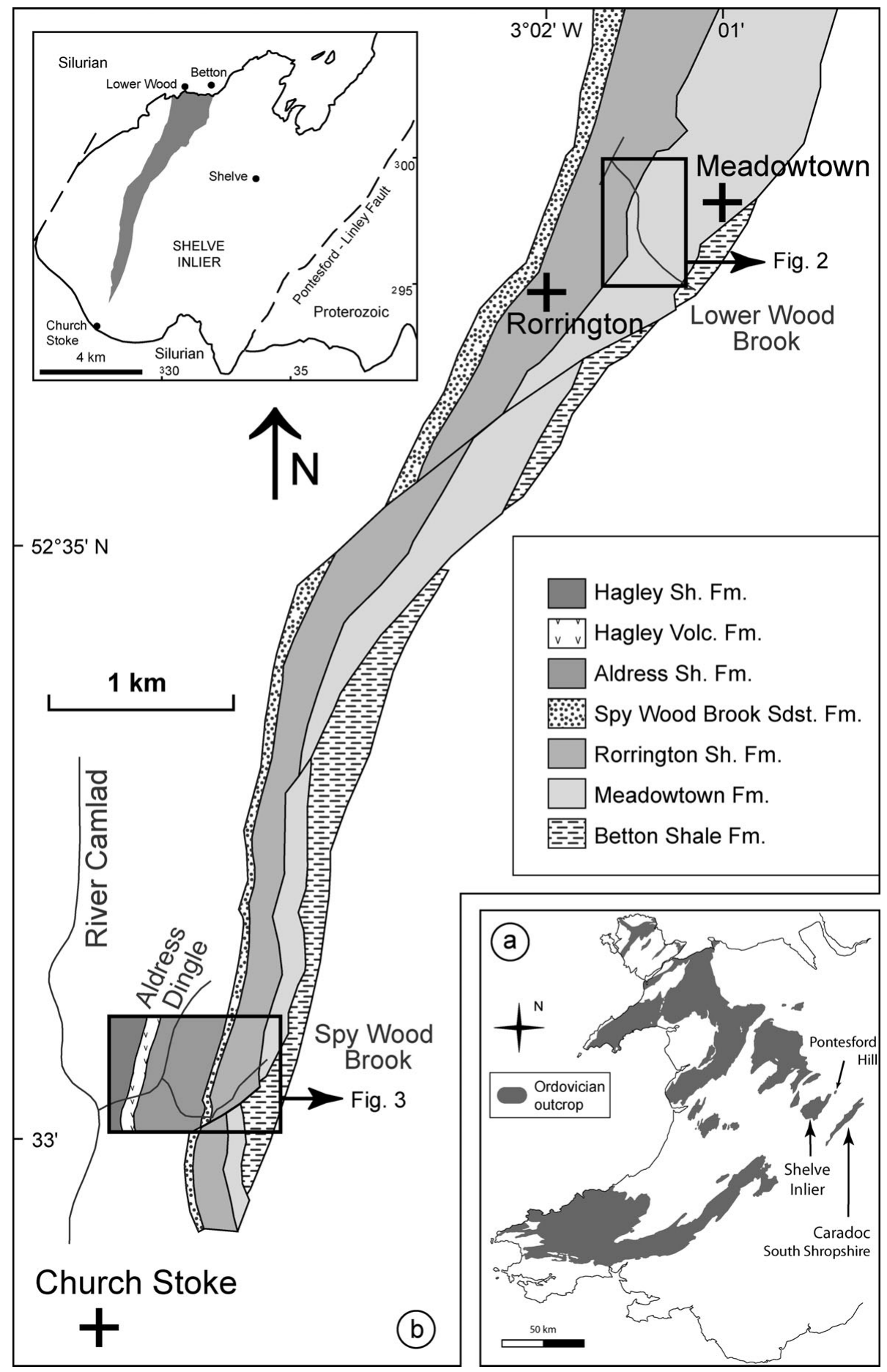

Figure 1. (a) Ordovician outcrops in Wales and the Welsh Borderland. (b) Location of the sections and summary geological map of the most relevant units in the Shelve Inlier (modified after Bettley, unpub. Ph.D. thesis, Univ. Oxford, 1998 and Cave \& Hains, 2001). The inset map has UK national grid coordinates: grid SJ in the north, grid SO in the south.

thesis, Univ. Oxford, 1998) reviewed the former faunas and focused on carefully measured sections containing mixed trilobite-graptolite faunas, among others in the Shelve area, and on their high-resolution correlation. Following the latter study, Bettley, Fortey \& Siveter (2001) proposed the Lower Wood Brook Section (Fig. 2) of the northern part of the Shelve Inlier as a possible type section for the base of the Nemagraptus gracilis Biozone. This is one of the reasons why we studied this section for chitinozoans, together with the additional Spy Wood Brook section (Fig. 3), although the section has never been officially brought to a vote before the International Subcommission on Ordovician Stratigraphy (ISOS) or the International Union of Geological Sciences (IUGS). The work by Bettley (unpub. Ph.D. thesis, Univ. Oxford, 1998) and Bettley, Fortey \& Siveter (2001) provides a calibration of our data with the other fossil groups present.

As far as previous chitinozoan research is concerned, the work of Jenkins (1967) has become a standard, 


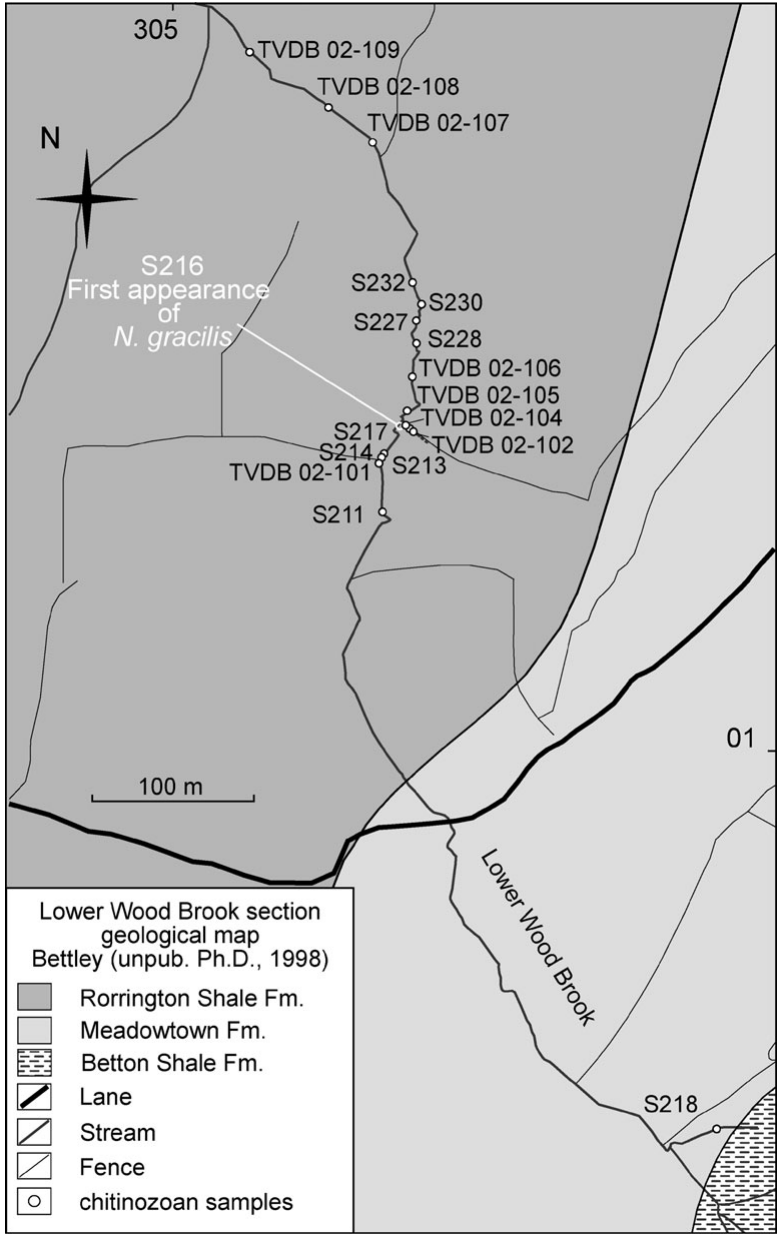

Figure 2. Geological map of the Lower Wood Brook section with the sample localities (after R. M. Bettley, unpub. Ph.D. thesis, Univ. Oxford, 1998). The map has UK national grid coordinates (see Fig. 1).

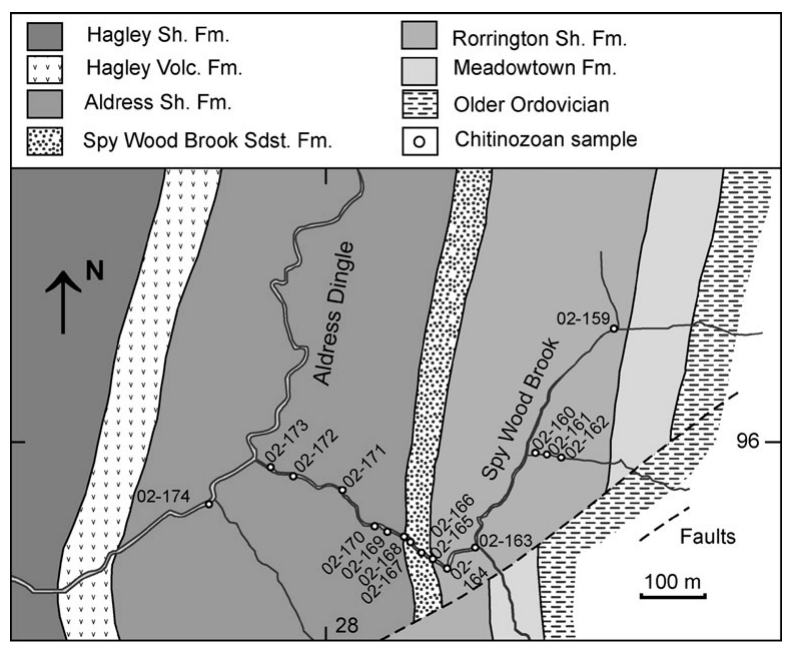

Figure 3. Geological map of the Spy Wood Brook section with the sample localities (after Hughes, 1989). The map has UK national grid coordinates (see Fig. 1).

and focused on Llanvirn and Caradoc chitinozoans, respectively, from the Shelve Inlier and the type Caradoc area (see below). The BGS (British Geological Survey) memoir for the Montgomery area (sheets
165/151) by Cave \& Hains (2001) is used here as the lithostratigraphical reference, upgrading most of Whittard's (1979) members to formations (as originally suggested by Lynas, 1985).

\section{1.c. The type Caradoc area}

The Caradoc succession in south Shropshire crops out south of the Church Stretton Fault, from Harnage in the north to Coston in the south, in two tracts that are separated by upfaulted Cambrian and Proterozoic rocks near Hope Bowdler and Cardington (Fig. 4). The sequence in the northern part is thicker, but the sequence in the south is more complete (Fortey et al. 2000; Rushton et al. 2000). As already mentioned, and in contrast to the Shelve area deposits at the far side of the Pontesford-Linley Fault, only the Caradoc Series is exposed in south Shropshire, where its basal beds rest diachronously and unconformably on rocks of Precambrian to Tremadoc age. The rocks are shallow-water deposits and mainly comprise sandstones, siltstones, mudstones and shales (Williams et al. 1972).

Dean (1958) reviewed the scientific contributions on the area, starting from Murchison's definition of the 'Caradoc Sandstone' (1839), which already postulated its best section along the Onny River as the 'type area'. The area was where Bancroft (1933) originally defined the subdivision of the Caradoc into seven stages, largely based on brachiopod biostratigraphy. Dean (1958, $1960,1964)$ reviewed the stages and lithostratigraphical units, and added the trilobite biostratigraphy to the schemes. For the lower units of the successions, he applied a separate lithostratigraphical terminology for the areas south and north of the Cardington area. The succession in the southern (Coston) part comprises the following formations, from bottom to top: the Coston, Smeathen Wood, Glenburrell, Horderley Sandstone, Alternata Limestone, Cheney Longville, Acton Scott and Onny formations (Dean, 1958; Fig. 5). In the northern (Chatwall) part, the succession consists of the Hoar Edge Grits, Harnage Shales, Chatwall Flags, Alternata Limestone, Cheney Longville and Acton Scott formations (Dean, 1960). This double terminology persists in more recent contributions (e.g. Williams et al. 1972; Savage \& Bassett, 1985; Rushton et al. 2000; Fortey et al. 2000). Some authors, though, seem to prefer using certain of the northern formation names in the southern part (e.g. Turner, 1982; A. Ancilletta, unpub. DEA thesis, Univ. Liège, 1997). We will follow Fortey et al. (2000). The areas with separate lithostratigraphical divisions coincide with the Cressage-Cardington and Onny sub-basins suggested by Smith \& Rushton (1993) (Rushton et al. 2000; Fortey et al. 2000).

It should be stressed that the Caradoc succession is incomplete in its type area. Apart from the already cited unconformity at the base of the beds, the Llandovery lies unconformably on the locally youngest Caradoc. This can be seen clearly in the famous cliff section in 


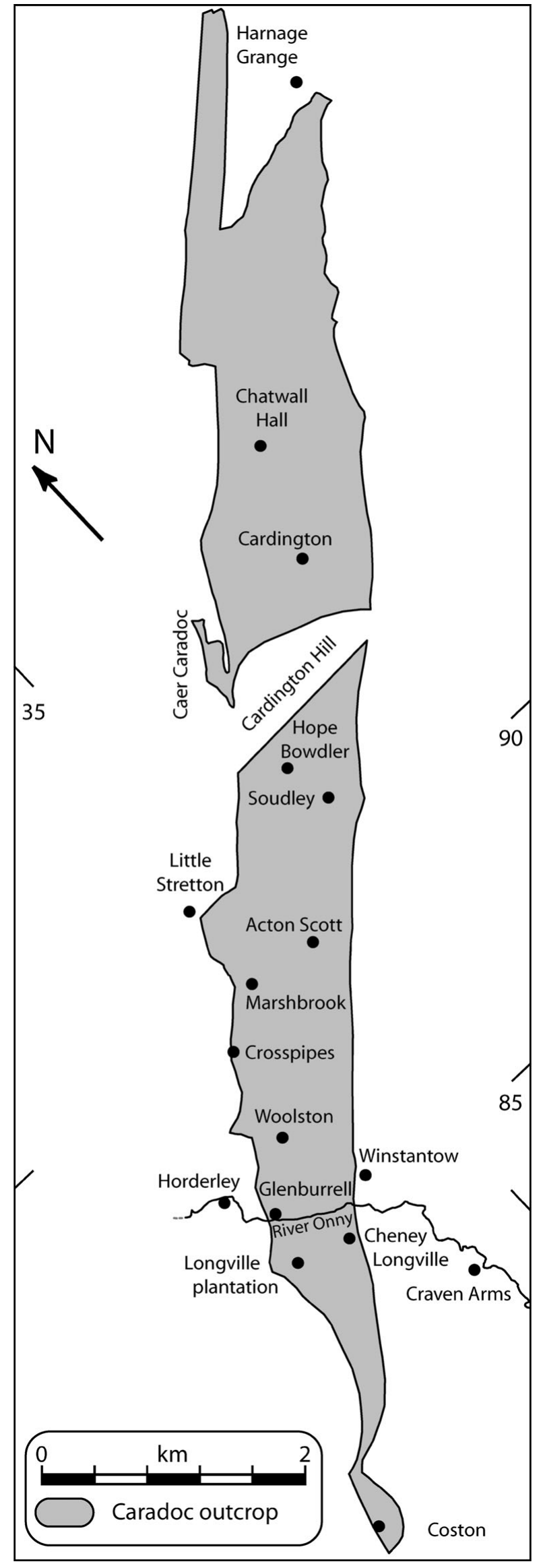

Figure 4. The Caradoc Inlier: sketch map of the northern and southern tracts of the type Caradoc area, showing the main localities and the Onny Valley section (modified after Dean, 1958). The national grid coordinates are from grid SO. the Onny Valley, where the Onnian rocks at the top of the Caradoc succession are overlain by the upper Llandovery Hughley Shale Formation with a very slight angular unconformity.

Fortey et al. (1995) downgraded the stages erected by Bancroft (1933; see also Dean, 1958 and Hurst, 1979) to substage level and contracted them into four stages 'of greater utility in both Anglo-Welsh and international correlation' (Fortey et al. 1995, p. 20). Further detailed information on chronostratigraphy, shelly faunas and lithostratigraphy can be found in most of the above-cited publications.

Nemagraptus gracilis has been recognized from the Hoar Edge Grits, in the Costonian substage (Pocock et al. 1938; Dean, 1958; Fortey et al. 2000; Rushton et al. 2000). Although graptolites from the higher stratigraphical levels, deposited in shallow water, are sparse, Dean (1958 and in Williams et al. 1972) drew the base of the Diplograptus multidens Biozone in the top part of the Costonian Stage (as in the Spy Wood Sandstone Formation: Williams et al. 1972, p. 40). He postulated that the Actonian Stage and most of the Onnian Stage belong to the Dicranograptus clingani Biozone. Rushton et al. (2000) suggested that the base of the $D$. multidens Biozone lies very close to the Costonian-Harnagian boundary, again based on correlations with Spy Wood Brook. Both Rushton et al. (2000) and Fortey et al. (2000) did not use the stratigraphically higher graptolite occurrences in their correlation schemes.

Savage \& Bassett (1985) reported conodonts from most of their samples taken from the south Shropshire Caradoc. They are rarely abundant and comprise no species diagnostic for biozones.

Turner (1982) reported Caradoc acritarchs from the type Caradoc area, mixed with reworked species of Tremadoc and Arenig/Llanvirn age, in an essentially inverted succession, illustrating successive erosion of progressively older source material. The peak of reworking seems to be located from halfway up the Horderley Sandstone Formation to midway in the Cheney Longville Formation (Turner, 1982, textfig. 5, p. 136), coinciding with energetically higher depositional conditions.

Jenkins (1967) studied the Caradoc chitinozoans from the southern Caradoc area, mainly from the Onny Valley section and its immediate vicinity. $\mathrm{He}$ recognized four separate chitinozoan assemblages, numbered 1 to 4 . This four-fold subdivision was conveniently used by Fortey et al. (1995), together with other fossil groups displaying a comparable pattern, to corroborate their division into four stages of the Caradoc. However, the stage boundaries do not precisely correspond to those of the chitinozoan assemblages. Thirty years later, Ancilletta (unpub. DEA thesis, Univ. Liège, 1997) restudied the systematics of the rich chitinozoan assemblages from the Onny Valley section using the scanning electron microscope (SEM), a technique unavailable to Jenkins. 


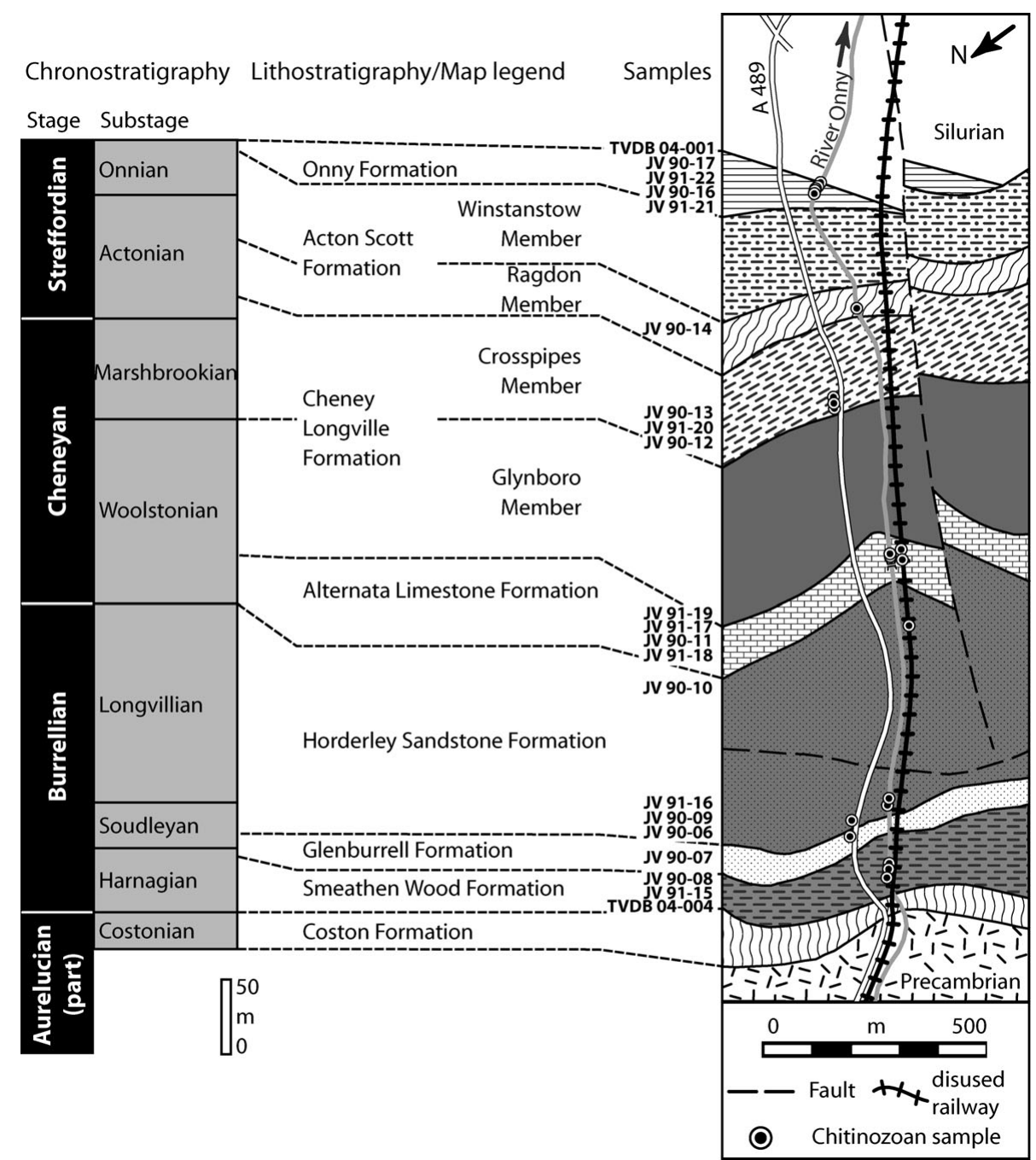

Figure 5. Geological map of the Onny Valley section with the sample localities and the chrono- and lithostratigraphy of the southern Caradoc area (after Rushton et al. 2000). For location of the section, see Figure 4.

\section{The sections studied, chitinozoan sampling and methodology}

\section{2.a. Shelve Inlier}

The Shelve Inlier section most important to our project is the Lower Wood Brook Section. It can be found between Rorrington and Meadowtown, from grid reference SJ 30910064 at the head of the stream to SJ 3055 0153, before the stream changes direction and continues along strike (R. M. Bettley, unpub. Ph.D. thesis, Univ. Oxford, 1998; Fig. 2). The section exposed consists of three formations, from bottom to top: the Betton Shale Formation, the Meadowtown Formation and the Rorrington Shale Formation and is unfaulted except for a normal fault at the base of the section (R. M. Bettley, unpub. Ph.D. thesis, Univ. Oxford, 1998).

The lithological appearance of the Betton Shale Formation is discussed by Whittard (1979) and by Cave \& Hains (2001, pp. 27-8); only the uppermost strata are exposed in the section.

The fauna and detailed lithology of the sand-, silt- and (dominating) mudstones of the Meadowtown Formation were described by Whittard (1979), Bettley (unpub. Ph.D. thesis, Univ. Oxford, 1998) and by Cave \& Hains (2001, pp. 28-30). Bettley attributed the entire formation to the Hustedograptus teretiusculus Biozone and to two successive trilobite biozones, namely, the Whittardolithus inopinatus and the Lloydolithus lloydii biozones (see Bettley, Fortey \& Siveter, 2001).

The Rorrington Shale Formation consists of blueblack shales with abundant graptolites (Whittard, 1979; Cave \& Hains, 2001). Complete faunal lists and logs were given by R. M. Bettley in an unpublished Ph.D. thesis (Univ. Oxford, 1998). The FAD (First Appearance Datum) of Nemagraptus gracilis is determined at a level $262.28 \mathrm{~m}$ above the base of the section, or $76 \mathrm{~m}$ above the base of the Rorrington Shale Formation, at locality S216 [SJ 3059 0128]. The section has subsequently been proposed as type section for the base of the $N$. gracilis Biozone and D. irregularis Subbiozone (Bettley, Fortey \& Siveter, 2001, pp. 945-6). The level represents the zonal boundary between the Hustedograptus teretiusculus and N. gracilis biozones, the Llanvirn-Caradoc boundary in the UK, or the global Middle-Upper Ordovician boundary. The base of the $N$. gracilis Biozone is drawn at about the 
same level as Hughes (1989) suggested, but higher than supposed earlier, by Williams et al. (1972), among others. Near the top of the section, the base of the Marrolithoides anomalis trilobite Biozone can be recognized. The upper part of the formation is unexposed.

A second section studied in the Shelve Inlier is found along Spy Wood Brook, a tributary to the Aldress Dingle, which in turn runs into the River Camlad (Figs 1, 3). Spy Wood Brook and the Aldress Dingle are Sites of Special Scientific Interest (SSSIs). In ascending order, the succession exposed consists of the Meadowtown, Rorrington Shale, Spy Wood Sandstone, Aldress Shale, Hagley Volcanic and Hagley Shale formations, all of which were studied in detail by Cave \& Hains (2001) and Whittard (1979). Bettley's research (unpub. Ph.D. thesis, Univ. Oxford, 1998) on this particular section was, like our own, restricted to the upper Rorrington Shale, Spy Wood Sandstone and Aldress Shale formations. He positioned the base of the Diplograptus foliaceus Biozone at the FAD of Orthograptus apiculatus in the Rorrington Shale Formation at $7.04 \mathrm{~m}$ below the base of the Spy Wood Sandstone Formation, and proposed the Spy Wood Brook section as the type section for this level.

Chitinozoan samples from the Shelve Inlier have been obtained on two occasions. A first batch of samples consists of graptolite slabs collected by Richard Bettley, nicely positioned vis-à-vis his measured sections and graptolite zonal boundaries. The samples are numbered $\mathrm{S} 2{ }^{* *} / \mathrm{slab}$ number. The slab number is irrelevant to our (destructive) approach and has been omitted in most cases below. Additional samples from Lower Wood Brook were collected in the field during the summer of 2002, using the maps provided by Richard Bettley (Figs 1,2). The samples are numbered TVDB $02-1^{* *}$ and are especially closely spaced across the base of the Nemagraptus gracilis graptolite Biozone. The samples from the Spy Wood Brook (and its tributary Dead Man's Dingle), in the southern part of the area, were collected from the Rorrington Shale, Spy Wood Sandstone and Aldress Shale formations, during the same field season, using the maps of Bettley (unpub. Ph.D. thesis, Univ. Oxford, 1998) and Hughes (1989; Fig. 3). The same TVDB $02-1^{* *}$ label type is used.

For his chitinozoan analysis, Jenkins (1967) collected most of his Llanvirn material from the Shelve area and his Caradoc material from the type Caradoc area. We were mainly interested in the Llanvirn-Caradoc transition and Caradoc successions in the Shelve area. Hence, there is little overlap in sampling between Jenkins' work and our study, with the exception of Jenkins' highest sample from the Shelve area (S11), which has been included in our range chart (Fig. 6).

\section{2.b. Sampling and methodology at Onny Valley}

The samples available to this study were the same as those of Ancilletta (unpub. DEA thesis, Univ. Liège,
1997). They were collected from the Onny Valley exclusively, which is the type locality for the Actonian and Onnian substages, and a SSSI. The collection covers the exposed levels of the succession described above and illustrated in Figure 5 (Coston to Onny formations). All sampled localities are as far as possible related to the localities and levels used by Jenkins (1967) and Turner (1982). Additional samples were collected in the summer of 2004, especially in the topmost Onnian exposed. All localities are described in the Appendix. Figure 5 illustrates their geographical and stratigraphical positions.

The memoir of A. Ancilletta (unpub. DEA thesis, Univ. Liège, 1997) was never published formally, and hence is virtually unavailable to the scientific community. His data have therefore been (partially) incorporated herein, with his approval. However, several of his species identifications and systematics remarks have been revised by the first author. Furthermore, numerical tables illustrating the concentrations of chitinozoans in Ancilletta's memoir showed inconsistencies. Therefore we considered only the absolute number of specimens recorded for each species (A. Ancilletta, unpub. DEA thesis, Univ. Liège, 1997, p. 44, table 2), as being accurate. As for Jenkins (1967), he did not record absolute frequencies. Keeping these difficulties in mind, our biostratigraphical study mainly focused on the re-evaluation of presence or absence of chitinozoan species, rather than their absolute concentration or the number of specimens per gram of rock.

Four of Ancilletta's samples (unpub. DEA thesis, Univ. Liège, 1997) were completely reinvestigated, including dissolution of new rock material according to standard palynological techniques. This allowed us to check the composition of the fauna and the absolute frequency of species. The samples treated as such include JV 90-07, 90-09, 91-16 and 90-13 and these were added to the samples collected in 2004, TVDB 04-001 and 04-004 (see Section 4). Topmost sample 04-001 was specifically taken in a futile attempt to recognize faunas from the CaradocAshgill transition as reported from northern England (see Vandenbroucke, Rickards \& Verniers, 2005). Additional specimens, to check identifications, were obtained from several of Ancilletta's stored residues (samples JV 90-12, 90-14, 90-16, 91-22 and 90-17), but it is not known how much of the residue originally obtained they represent (see Section 4).

\section{Chitinozoan results from the Shelve Inlier}

Twenty-one samples from the Lower Wood Brook section and fifteen samples from the Spy Wood Brook section have been processed for chitinozoans. Most samples yielded a high number of moderately to well-preserved chitinozoans. The results of the chitinozoan study in the Shelve area are shown qualitatively on Figures 6, 7 and 8, quantitatively on Figures 9 and 10, and briefly discussed below, by 


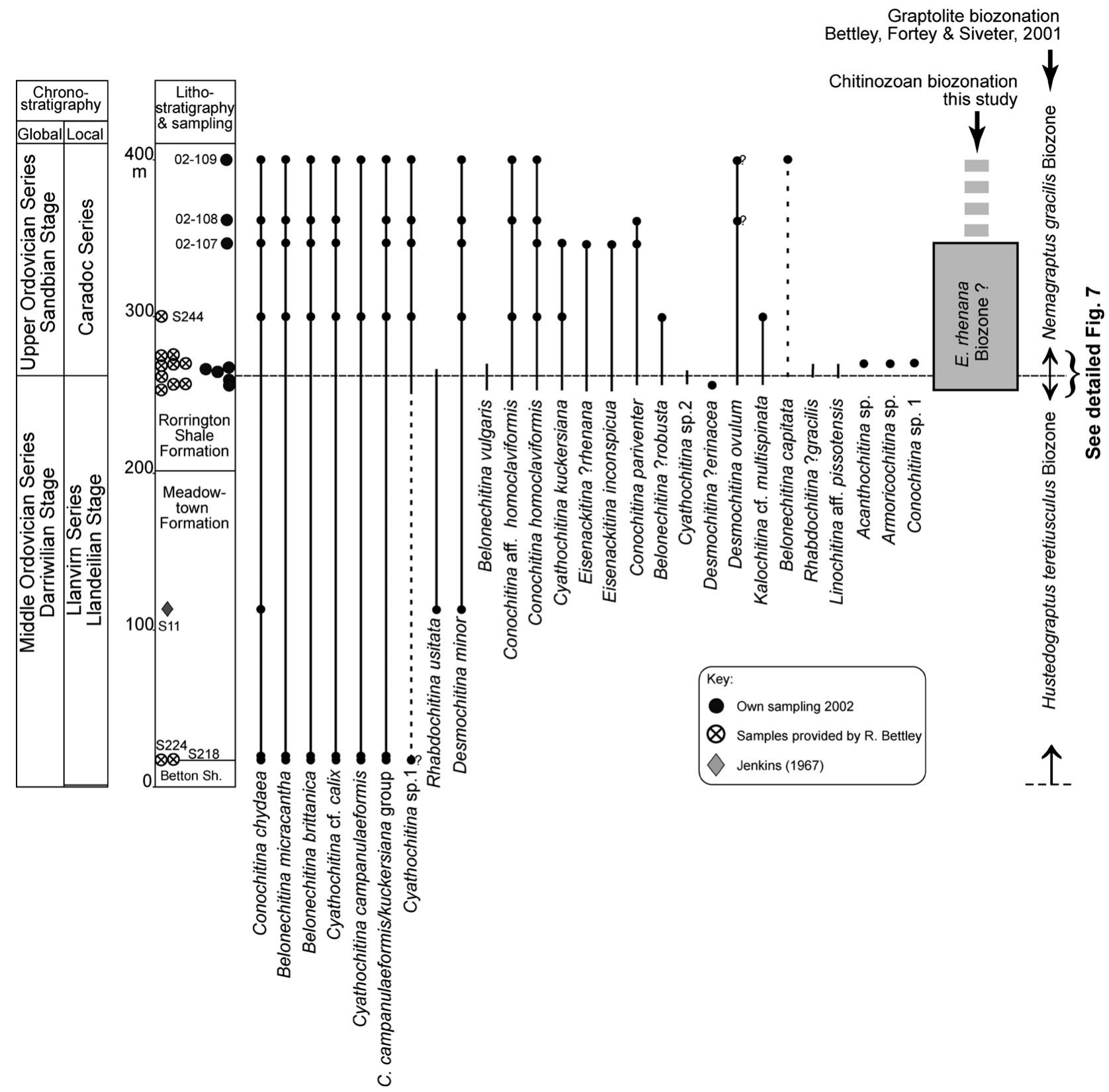

Figure 6. Range chart of chitinozoan species in the Lower Wood Brook section (Shelve Inlier).

section and in ascending stratigraphical order, with emphasis on the FADs of the particular species. A systematic review of the chitinozoans from the study areas is undertaken in a Palaeontographical Society Monograph by Vandenbroucke (2008b).

\section{3.a. Lower Wood Brook}

The two lowermost samples, stratigraphically more than $200 \mathrm{~m}$ below the base of the Nemagraptus gracilis Biozone, yielded long-ranging species Conochitina chydaea, Belonechitina micracantha, Belonechitina brittanica, Cyathochitina cf. calix, Cyathochitina campanulaeformis, Cyathochitina campanulaeformiskuckersiana group, in addition to some poorly identified Cyathochitina sp. 1. These forms occur in almost every sample (see Fig. 6). Preservation in these two samples is rather poor.

All hitherto cited species continue to occur in great numbers higher up, throughout the samples taken around the lower boundary of the Nemagraptus gracilis graptolite Biozone as shown on the detailed range chart in Figure 7. They are joined by the common species Conochitina homoclaviformis and Conochitina aff. homoclaviformis. Desmochitina minor and Belonechitina vulgaris appear at the same level. Eisenackitina ?rhenana and Eisenackitina inconspicua range from sample S211 upwards, usually in lower numbers and in fewer samples than most of the other species.

Conochitina parviventer, Belonechitina ?robusta, Cyathochitina sp. 2, Desmochitina ovulum, Desmochitina ?erinacea and Kalochitina cf. multispinata can be found from sample TVDB 02-102 upwards, except 


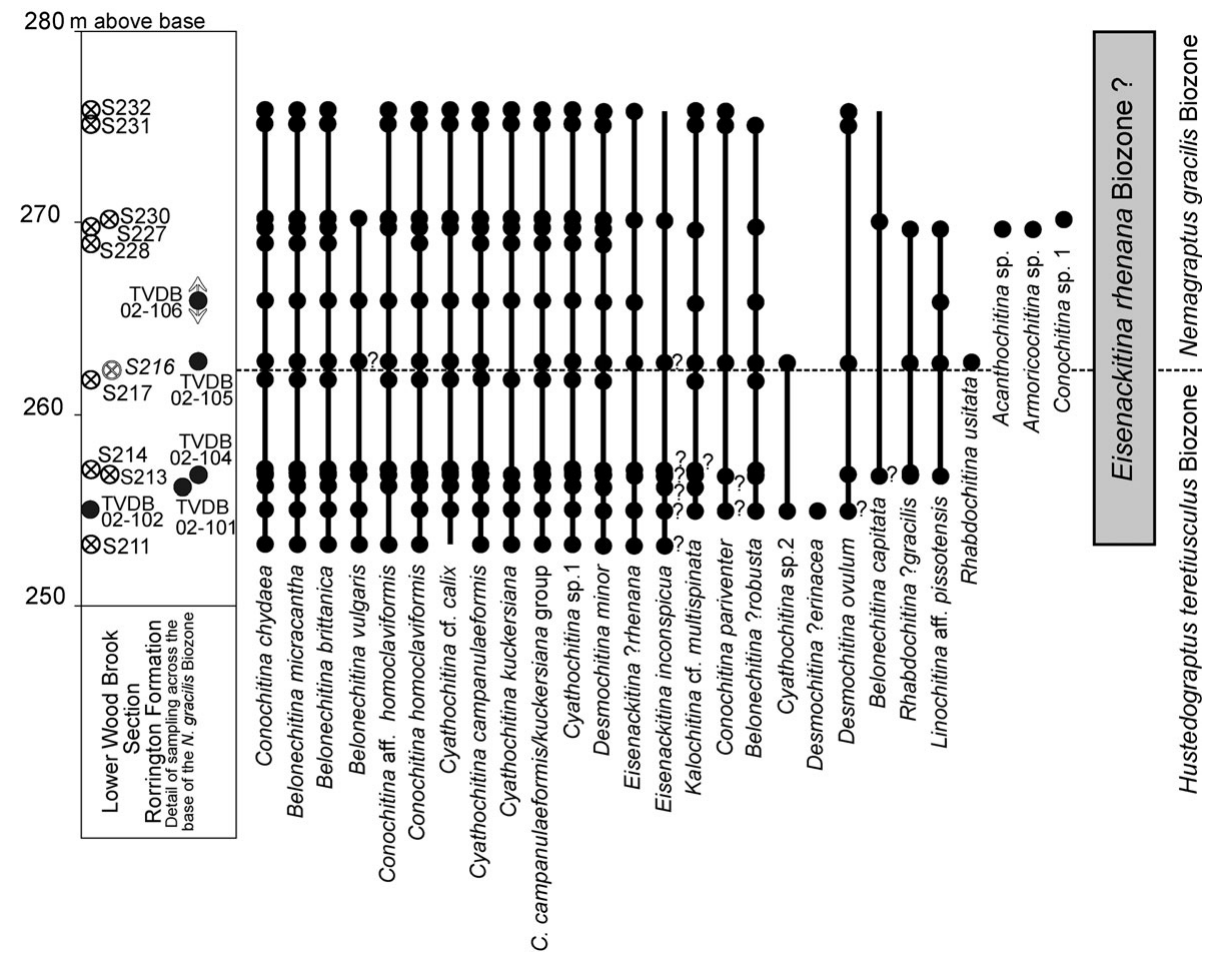

Figure 7. Detailed range chart of chitinozoan species across the base of the Nemagraptus gracilis Biozone, or the base of the global Upper Ordovician Series or of the British Caradoc Series in the Lower Wood Brook section (Shelve Inlier). For legend see Figure 6.

for $D$. ?erinacea, which is restricted to sample 02-102.

Linochitina aff. pissotensis and doubtful observations of Belonechitina capitata and Rhabdochitina ?gracilis are reported from slightly higher up-section onwards (sample TVDB 02-104). From more or less the same level, Laufeldochitina fragments have been reported on previous accounts by Vandenbroucke et al. (2003), but further observations discovered that this identification could not be sustained due to poor preservation.

Single sample observations of one specimen of the genus Acanthochitina, two belonging to Armoricochitina, one Rhabdochitina usitata, and of several specimens of Conochitina sp. 1 are listed in Figure 9.

\section{3.b. Spy Wood Brook}

Samples from the Rorrington Shale Formation in Spy Wood Brook yield no new species in comparison to the results obtained from Lower Wood Brook, with exception of a few observations of Fungochitina aff. actonica in samples TVDB 02-160 and 02-164. The same is true for samples taken from the finergrained horizons within the Spy Wood Sandstone Formation; worthwhile mentioning might be the first occurrence in this section of Eisenackitina ?rhenana in sample TVDB 02-167 (ranging up to TVDB 02172). Apart from the quite peculiar Belonechitina sp. 1 in sample TVDB 02-168, the lower samples from the Aldress Shale Formation continue to yield species already reported lower in the section or from Lower
Wood Brook. Somewhat higher up-section, new species appear, represented by many specimens in open nomenclature, in sample TVDB 02-171 (Hercochitina spp., Hercochitina aff. frangiata and a single Acanthochitina specimen). In sample TVDB 02-172, there is quite an influx of species previously not seen, such as Conochitina tigrina, Cyathochitina sp. 3, Euconochitina cf. conulus, Lagenochitina aff. dalbyensis, Lagenochitina sp. A aff. capax, Rhabdochitina magna, Spinachitina bulmani and Siphonochitina robusta, together with a rather complete set of species from the long-ranging assemblage cited above.

\section{Chitinozoan results from the Onny Valley}

Figure 11 lists the results of the samples we studied. Figure 12 gives an overview of the data obtained from the three chitinozoan studies in the area. Selected samples from Jenkins (1967, table I, p. 482) are also included, which are not from the Onny Valley section itself. Rather than discussing the results bed by bed or level by level, the most important points of discrepancy with the studies of Jenkins (1967) and Ancilletta (unpub. DEA thesis, Univ. Liège, 1997) are listed below; many of those are discussed in detail by Vandenbroucke (2008b). The latter publication also gives the formal description of all the species from this section. Selected ranges are shown in Figure 13; a selection of species is shown in Figure 14.

(1) Jenkins (1967) reported Lagenochitina baltica with rare occurrences low in the section, and more 


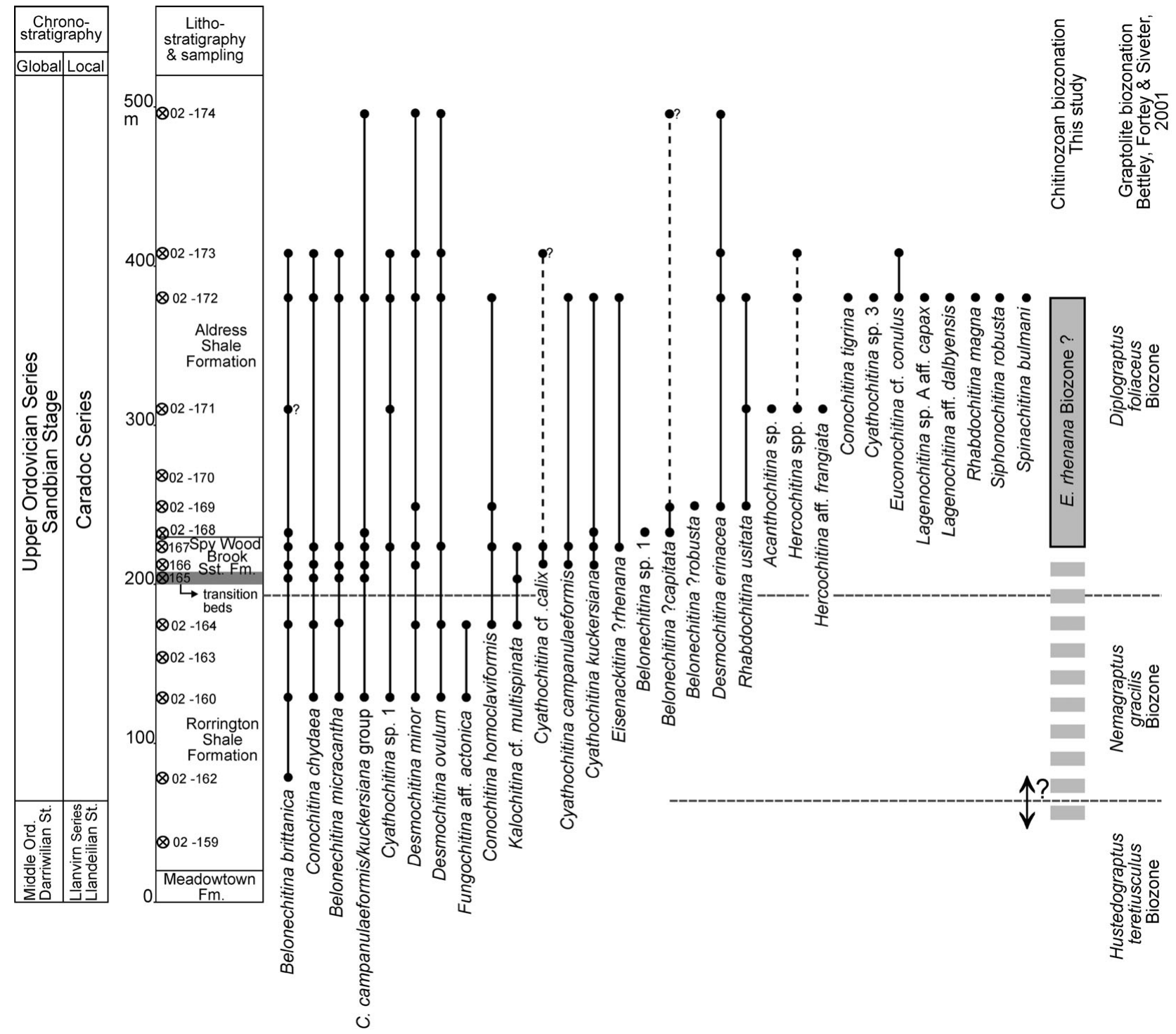

Figure 8. Range chart of chitinozoan species in the Spy Wood Brook section (Shelve Inlier). 'St.' - 'Stage'.

frequent ones in the Onny Formation. Ancilletta (unpub. DEA thesis, Univ. Liège, 1997) also found the species in the Onny Formation. In the samples and residues (re)studied in 2005, however, we did not find any L. baltica. Moreover, neither Jenkins' nor Ancilletta's photographs allowed recognition of the typical granular ornamentation of the species, although the latter author mentioned the granules in his description. Examining the specimens from the Jenkins collections, deposited at Sheffield University, we were unable to provide a decisive answer to the uncertainty concerning the species' presence in the Onny Valley section; the specimens of the Onny Formation (Jenkins' C1 levels) might indeed be attributed to L. baltica, although we remain cautious without SEM observation of the ornamentation. However, we have serious doubts about the identification of the (fragmentary) ones from the Glenburrell Formation (Jenkins' C11, Burrellian), which most probably are another species. In addition to this morphological uncertainty, the species occurs aberrantly low in the Onny Valley stratigraphy (in Jenkins, 1967), while in other sections it does not range below the base of the Fungochitina spinifera Biozone (Onnian to Cautleyan in northern England).

However, the samples (re)studied in 2005 confirm the presence of Lagenochitina prussica (see Figs 11, 12) in the Onny Formation, as suggested by Ancilletta (unpub. DEA thesis, Univ. Liège, 1997). L. prussica and L. baltica are morphologically similar; L. prussica differs from $L$. baltica only by its more spherical chamber shape, and is the only contemporaneous $L a$ genochitina species that bears the same characteristic ornamentation. Both are known to co-occur, the FAD of $L$. prussica only slightly later than that of $L$. baltica (Nõlvak \& Grahn, 1993). In short, we are quite sceptical about the rare, lower occurrences of L. baltica mentioned by Jenkins (1967), but the records of the species and of $L$. prussica from the Onny Formation seem to be valid.

(2) Spinachitina multiradiata from the Onny Valley has large basal spines and is systematically close to 


\begin{tabular}{|c|c|c|c|c|c|c|c|c|c|c|c|c|c|c|c|c|c|c|c|c|c|c|}
\hline $\begin{array}{c}\text { Chitinozoan results from the Lower Wood } \\
\text { Brook section }\end{array}$ & $\begin{array}{l}\bar{\Sigma} \\
\bar{\infty} \\
\omega \\
\omega\end{array}$ & $\begin{array}{l}\infty \\
\underset{N}{+} \\
\underset{N}{+}\end{array}$ & $\underset{\infty}{\stackrel{N}{\Sigma}}$ & 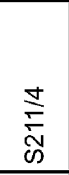 & 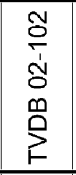 & 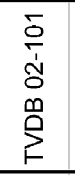 & 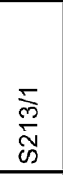 & $\begin{array}{l}\frac{N}{N} \\
\stackrel{N}{N}\end{array}$ & $\underset{\aleph}{*}$ & 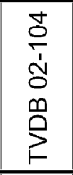 & $\begin{array}{l}\underset{\Sigma}{\Sigma} \\
\underset{\infty}{\Sigma}\end{array}$ & 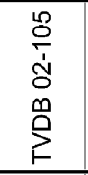 & 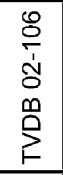 & 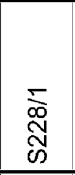 & $\begin{array}{l}\mathbb{N} \\
\text { N } \\
\text { d }\end{array}$ & $\begin{array}{l}\text { ల్ల్ } \\
\text { ర్ }\end{array}$ & $\begin{array}{l}\stackrel{\oplus}{\sim} \\
\underset{N}{N} \\
\tilde{\omega}\end{array}$ & $\begin{array}{l}\text { స్ల్ } \\
\text { ల్ }\end{array}$ & \begin{tabular}{l}
\multirow{J}{*}{} \\
J
\end{tabular} & 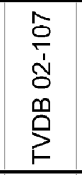 & $\begin{array}{l}0 \\
\stackrel{0}{1} \\
\dot{1} \\
0 \\
0 \\
0 \\
0 \\
\end{array}$ & 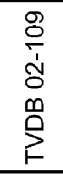 \\
\hline Chitinozoa indet. & 84 & 27 & 37 & 34 & \begin{tabular}{|l|}
201 \\
\end{tabular} & 105 & 33 & 35 & 60 & 156 & 64 & 68 & 76 & 35 & 62 & 52 & 48 & 33 & 41 & $34+37 \approx$ & 20 & 33 \\
\hline Belonechitina spp. & 2 & & & & & & 1 & & & & & & & 1 & & 2 & & & 1 & & & \\
\hline Conochitina chydaea & 107 & 106 & 11 & 5 & 39 & 8 & 24 & 23 & 17 & 10 & 2 & 3 & 41 & 16 & 24 & 79 & 24 & 4 & 31 & 117 & 5 & 10 \\
\hline Belonechitina aff. brittanica & 8 & 5 & 1 & & 35 & 3 & 8 & 10 & 9 & 47 & 9 & 19 & 24 & 9 & 15 & & 14 & 5 & 1 & 8 & & \\
\hline Belonechitina brittanica & 57 & 4 & 9 & 29 & 359 & 99 & 88 & 79 & 156 & 278 & 72 & 598 & 359 & 24 & 83 & 12 & 40 & 37 & 20 & 1 & 17 & 9 \\
\hline Belonechitina micracantha & $21(+? 13)$ & 7 & 28 & 11 & 110 & 29 & 22 & 20 & 42 & $56+? 2$ & 2 & $36+? 1$ & 7 & 19 & 5 & 83 & 15 & 6 & 45 & 266 & 3 & 12 \\
\hline Cyathochitina of. calix & 20 & 4 & & & 55 & 5 & 11 & 13 & 12 & 54 & 3 & 17 & 5 & & 11 & 13 & 17 & 3 & 28 & 1 & 4 & 16 \\
\hline Cyathochitina campanulaeformis & 20 & 3 & 6 & 5 & 12 & 21 & & & 22 & 11 & 4 & 2 & 7 & 6 & 16 & 7 & 5 & 1 & 8 & & & 1 \\
\hline Cyathochitina campanulaeformis/kuckersiana group & 18 & 3 & 6 & 10 & 130 & 10 & 35 & 30 & 39 & 122 & 16 & 82 & 47 & 24 & 12 & 60 & 20 & 13 & 23 & 26 & 9 & 18 \\
\hline Cyathochitina sp. 1 & $8 ?$ & & 9 & 14 & 77 & 15 & 15 & 14 & 26 & 74 & 19 & 104 & 35 & 9 & 32 & 26 & 7 & 8 & 4 & 19 & 2 & 9 \\
\hline Cyathochitina spp. & 15 & 1 & 3 & 11 & 71 & 26 & & 8 & 28 & 70 & 4 & 7 & 14 & 17 & 12 & 24 & 13 & 4 & 13 & 9 & 5 & 14 \\
\hline Eisenackitina spp. & 4 & 2 & & & 3 & & & & & & & & & & 1 & 7 & 3 & 3 & & & & \\
\hline Rhabdochitina spp. & 6 & & & 3 & 2 & 4 & & & 4 & 25 & 3 & & 14 & 1 & & 3 & & 2 & & & 4 & 6 \\
\hline Belonechitina vulgaris & & & 14 & 4 & 6 & & & & $16+? 2$ & 13 & & $? 5$ & $2+? 1$ & & & 1 & & & & & & \\
\hline Conochitina homoclaviformis & & & 33 & 56 & 49 & 235 & 30 & 26 & 26 & 323 & 123 & 201 & 145 & 8 & 66 & 2 & 13 & 32 & 27 & 8 & 42 & 15 \\
\hline Desmochitina minor & & & 2 & 2 & 21 & 10 & 31 & 5 & 4 & 7 & 16 & 176 & 6 & 26 & 4 & 28 & 66 & 17 & 3 & 2 & 3 & 6 \\
\hline Eisenackitina ?henana & & & 4 & 2 & 3 & 1 & & 5 & 2 & 2 & & 2 & 1 & & & 11 & & 14 & & 48 & & \\
\hline Eisenackitina inconspicua & & & $? 2$ & $? 1$ & $5+? 2$ & $1+? 1$ & $? 1$ & 1 & $? 1$ & 6 & & $? 1$ & & & & 15 & & & & 3 & & \\
\hline Conochitina aff. homoclaviformis & & & & 6 & & 12 & & & 1 & 15 & 2 & 10 & 14 & & 5 & 3 & 5 & 5 & 20 & & 3 & 2 \\
\hline Cyathochitina kuckersiana & & & & 1 & 4 & 2 & & & 1 & 2 & 3 & & 1 & 13 & 4 & 27 & 5 & 6 & 2 & 6 & & \\
\hline Kalochitina cf multispinata & & & & & 26 & 13 & 10 & 4 & 9 & 33 & 10 & 25 & 16 & & 1 & & 1 & 1 & 1 & & & \\
\hline Conochitina parviventer & & & & & $? 5$ & & & & & $? 3$ & & 1 & & & & & 1 & 2 & & 5 & 1 & \\
\hline Belonechitina ?robusta & & & & & 2 & & 1 & & 1 & 3 & $P$ & 23 & 10 & & 1 & & 9 & & 1 & & & \\
\hline Cyathochitina sp. 2 & & & & & 1 & & & & & & & 2 & & & & & & & & & & \\
\hline Desmochitina ?erinacea & & & & & 1 & & & & & & & & & & & & & & & & & \\
\hline Desmochitina ovulum & & & & & $? 1$ & & & 1 & & & & 8 & & & & & $4+? 2$ & 2 & & & $? 1$ & $? 1$ \\
\hline Conochitina spp. & & & & & 3 & & & & 3 & & & & 1 & & 2 & & & 3 & & & & \\
\hline Lagenochitina spp. & & & & & & 1 & & & & 3 & & 1 & & & & 2 & & & & & & \\
\hline Rhabdochitina ?gracilis & & & & & & & 3 & 4 & & $1+1 ?$ & & 23 & & & 1 & & & & & & & \\
\hline Euconochitina spp. & & & & & & & & & 2 & & & & & & 1 & 3 & & & & & & \\
\hline Eisenackitina spp. & & & & & & & & & & 2 & & & & & & & & & 1 & 2 & & \\
\hline Belonechitina capitata & & & & & & & & & & $? 1$ & & & & & & 1 & & & & & & 1 \\
\hline Linochitina aff. pissotensis & & & & & & & & & & 3 & & 42 & 2 & & 1 & & & & & & & \\
\hline Rhabdochitina usitata & & & & & & & & & & & & 1 & & & & & & & & & & \\
\hline Acanthochitina sp. & & & & & & & & & & & & & & & 1 & & & & & & & \\
\hline Armoricochitina sp. & & & & & & & & & & & & & & & 2 & & & & & & & \\
\hline Conochitina sp. 1 & & & & & & & & & & & & & & & & 21 & & & & & & \\
\hline scolecodonts & & & & & & & $x$ & & & $x$ & & & & & & $x$ & $x$ & $x$ & & $x$ & & $x$ \\
\hline acritarchs & & & & & & & & & & & & & & & & $x$ & $x$ & & & & & \\
\hline total amount of chitinozoans & 383 & 162 & 165 & 194 & 1223 & 601 & 313 & 278 & 483 & 1323 & 352 & 1458 & 828 & 208 & 362 & 482 & 312 & 201 & 270 & 928 & \begin{tabular}{|c|}
119 \\
\end{tabular} & 153 \\
\hline amount of dissolved rock (g) & 12.11 & 14.79 & 13.54 & 13.98 & 10.78 & 10.47 & 6.62 & 5.22 & 12.85 & 10.67 & 12.77 & 10.04 & 10.66 & 13.60 & 13.53 & 13.61 & 13.18 & 13.10 & 13.30 & 10.35 & 10.43 & 10.91 \\
\hline percentage of the residue picked & 100 & 100 & 25 & 12 & 100 & 100 & 50 & 50 & 25 & 100 & 25 & c. 50 & 50 & 50 & 25 & 25 & 50 & 25 & 100 & 18 & 100 & 100 \\
\hline
\end{tabular}

Figure 9. Numerical results of the chitinozoan study in the Lower Wood Brook section of the Shelve Inlier. 


\begin{tabular}{|c|c|c|c|c|c|c|c|c|c|c|c|c|c|c|c|}
\hline $\begin{array}{l}\text { Chitinozoan results from the Spy Wood } \\
\text { Brook section }\end{array}$ & 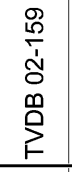 & 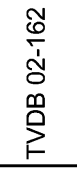 & 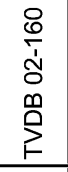 & 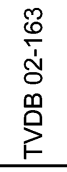 & 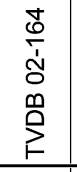 & 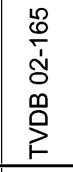 & 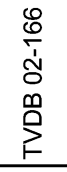 & 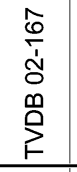 & 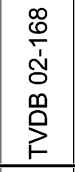 & 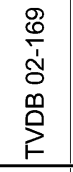 & 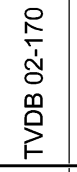 & 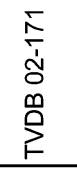 & 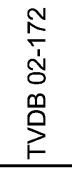 & 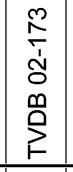 & 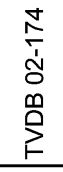 \\
\hline Chitinozoa indet. & & 6 & 34 & 7 & 84 & 34 & 61 & 55 & 55 & 45 & 1 & 57 & 87 & 25 & 17 \\
\hline Belonechitina brittanica & & 1 & 56 & & 157 & 85 & 35 & 228 & 36 & & & $? 1$ & 9 & 4 & \\
\hline Belonechitina aff. brittanica & & & 9 & & 7 & & 1 & 17 & & & & & & & \\
\hline Conochitina chydaea & & & 11 & & 3 & 2 & 3 & 6 & & & & & 25 & 2 & \\
\hline Belonechitina micracantha & & & 26 & & 9 & 2 & 1 & 2 & & & & & 25 & 1 & \\
\hline Conochitina spp. & & & 2 & & 9 & 1 & & & & & & & 3 & & \\
\hline Cyathochitina sp. 1 & & & 41 & & & & & 14 & & & & 3 & 2 & & \\
\hline Cyathochitina campanulaeformis/kuckersiana group & & & 17 & & 7 & 7 & 15 & 44 & 5 & & & & 4 & 1 & 6 \\
\hline Cyathochitina spp. & & & 1 & & & 7 & 1 & 3 & 3 & 1 & & 24 & 7 & & \\
\hline Desmochitina ovulum & & & 6 & & 19 & & & 1 & & 12 & & & 3 & 1 & 2 \\
\hline Desmochitina minor & & & 11 & & 125 & & 7 & 3 & & 60 & & & 8 & 6 & 4 \\
\hline Fungochitina aff. actonica & & & 2 & & 5 & & & & & & & & & & \\
\hline Rhabdochitina spp. & & & 2 & & 2 & & & 3 & & 1 & & & 9 & 1 & 1 \\
\hline Belonechitina spp. & & & & 1 & 1 & & 2 & 20 & 2 & 1 & & 5 & 8 & 2 & 10 \\
\hline Conochitina homoclaviformis & & & & & 59 & & & 21 & & 4 & & & 8 & & \\
\hline Kalochitina cf. multispinata & & & & & 2 & 2 & & 13 & & & & & & & \\
\hline Cyathochitina cf. calix & & & & & & 1 & 86 & & & & & & & $? 3$ & \\
\hline Cyathochitina campanulaeformis & & & & & & 2 & 3 & & & & & & 5 & & \\
\hline Cyathochitina kuckersiana & & & & & & 1 & 2 & 2 & & & & & 1 & & \\
\hline Eisenackitina spp. & & & & & & & & 1 & & & & & 1 & & \\
\hline Eisenackitina ?rhenana & & & & & & & & 1 & & & & & 8 & & \\
\hline Belonechitina ?capitata & & & & & & & & & 3 & $\mathrm{P}$ & & & & & $? 1$ \\
\hline Belonechitina sp. 1 & & & & & & & & & 21 & & & & & & \\
\hline Spinachitina spp. & & & & & & & & & 1 & 2 & 1 & 1 & & & \\
\hline Desmochitina erinacea & & & & & & & & & & 50 & & & 3 & 8 & 2 \\
\hline Belonechitina ?robusta & & & & & & & & & & $\mathrm{P}$ & & & & & \\
\hline Rhabdochitina usitata & & & & & & & & & & 47 & & $3+? 6$ & 4 & & \\
\hline Acanthochitina spp. & & & & & & & & & & & & 1 & & & \\
\hline Hercochitina spp. & & & & & & & & & & & & 13 & 3 & 1 & \\
\hline Hercochitina aff. frangiata & & & & & & & & & & & & 2 & & & \\
\hline Conochitina tigrina & & & & & & & & & & & & & 10 & & \\
\hline Cyathochitina sp. 3 & & & & & & & & & & & & & 3 & & \\
\hline Euconochitina cf. conulus & & & & & & & & & & & & & 12 & 3 & \\
\hline Euconochitina spp. & & & & & & & & & & & & & 3 & 1 & \\
\hline Lagenochitina aff. dalbyensis & & & & & & & & & & & & & 2 & & \\
\hline Lagenochitina sp. A aff. capax & & & & & & & & & & & & & 2 & & \\
\hline Lagenochitina spp. & & & & & & & & & & & & & 5 & & \\
\hline Rhabdochitina magna & & & & & & & & & & & & & 8 & & \\
\hline Spinachitina bulmani & & & & & & & & & & & & & 8 & & \\
\hline Siphonochitina robusta & & & & & & & & & & & & & 4 & & \\
\hline Tanuchitina spp. & & & & & & & & & & & & & 1 & & \\
\hline acritarchs & & & & $\mathrm{x}$ & & & & & & & & & & & \\
\hline Foraminifera & & & & $x$ & & & & & & & & & & & \\
\hline scolecodonts & & & & & $\mathrm{P}$ & $x$ & & $x$ & $\mathrm{x}$ & & & & & & \\
\hline total amount of chitinozoans & 0 & 7 & 218 & 8 & 489 & 144 & 217 & 434 & 126 & 223 & 2 & 115 & 281 & 59 & 43 \\
\hline amount of dissolved rock (g) & 10.05 & 10.12 & 10.52 & 10.92 & 10.84 & 10.38 & 10.58 & 10.19 & 10.37 & 10.70 & 10.85 & 15.13 & 49.56 & 10.27 & 10.67 \\
\hline percentage of the residue picked & 100 & 100 & 100 & 100 & 100 & 100 & 100 & 33 & 100 & 14 & 100 & 100 & 100 & 100 & 100 \\
\hline
\end{tabular}

Figure 10. Numerical results of the chitinozoan study in the Spy Wood Brook section of the Shelve Inlier.

the smooth Spinachitina cervicornis specimens, figured by Nõlvak \& Grahn (1993) to illustrate the index species of their eponymous biozone (see discussion in Section 5.b). Previous chitinozoan studies in the section (Jenkins, 1967; A. Ancilletta, unpub. DEA thesis, Univ. Liège, 1997) respectively identified the species as Ancyrochitina bulmani and Spinachitina bulmani. The species has a more extended range in our and Ancilletta's studies, compared to the range reported for $A$. bulmani by Jenkins.

(3) Acanthochitina pudica has been found on the same levels as by Ancilletta (unpub. DEA thesis, Univ. Liège, 1997), but apparently was not observed by Jenkins.
(4) Ancyrochitina alaticornis as reported by both Jenkins (1967) and Ancilletta (unpub. DEA thesis, Univ. Liège, 1997) has herein been attributed to a different genus and split into Spinachitina cervicornis and Spinachitina katherinae. The difference between the two species is that the latter undoubtedly bears ornamental crests on the vesicle wall. The original, smooth to lightly ornamented, A. alaticornis of Jenkins (1967) is considered synonymous to $S$. cervicornis, following practice in Baltoscandia (J. Nõlvak, pers. comm. 2007; Y. Grahn, pers. comm. 2006). Ancilletta's emendation of A. alaticornis to include heavily ornamented forms ( $S$. katherinae) is rejected. 


\begin{tabular}{|c|c|c|c|c|c|c|c|c|c|c|}
\hline $\begin{array}{l}\text { (Additional) chitinozoans from selected samples } \\
\text { from Onny Valley (Shropshire) }\end{array}$ & $\begin{array}{l}\text { ப் } \\
\text { o } \\
\dot{1} \\
\text { o } \\
0 \\
0 \\
\geq\end{array}$ & $\begin{array}{l}\text { 옹 } \\
\text { ò } \\
\text { o } \\
3\end{array}$ & $\begin{array}{l}\text { o } \\
\text { 잉 } \\
\text { o } \\
3\end{array}$ & $\frac{0}{\frac{1}{\sigma}}$ & $\begin{array}{l}\frac{N}{1} \\
\text { \& } \\
3\end{array}$ & 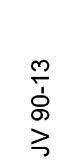 & $\begin{array}{l}\frac{\nabla}{1} \\
\text { ò } \\
3\end{array}$ & $\begin{array}{l}\text { 호 } \\
\text { ᄋ̀ } \\
3\end{array}$ & $\begin{array}{l}\underset{N}{N} \\
\stackrel{\sigma}{3}\end{array}$ & $\begin{array}{l}\overline{8} \\
0 \\
\dot{1} \\
0 \\
0 \\
\stackrel{0}{\gtrless}\end{array}$ \\
\hline Chitinozoa indet. & 85 & 12 & 16 & 16 & 14 & & 34 & 11 & 2 & 39 \\
\hline Belonechitina brittanica & 8 & & & & 1 & & & & & \\
\hline Belonechitina robusta & $9 ?$ & $2 ?$ & 9 & 8 & $1 ?$ & 5 & & & & \\
\hline Conochitina chydaea & 8 & 1 & 20 & & 6 & & & 2 & & \\
\hline Conochitina minnesotensis & 11 & 1 & 4 & 4 & 1 & & & 1 & & \\
\hline Conochitina ?tigrina & 1 & & & & & & & & & \\
\hline Conochitina sp. 2 & 3 & & & 2 & & & & & & \\
\hline Conochitina spp. & 9 & & 5 & 7 & 1 & & 3 & 9 & 1 & \\
\hline Cyathochitina campanulaeformis & 6 & 1 & 32 & 51 & 1 & & & 1 & & \\
\hline Cyathochitina cf. calix & 9 & & 2 & 4 & & & & & & \\
\hline Cyathochitina kuckersiana & 1 & 15 & 31 & 6 & & & 17 & 55 & 41 & 97 \\
\hline Cyathochitina sp. 1 & 2 & & & & & & & & & \\
\hline Cyathochitina spp. & 6 & 2 & 22 & 3 & 2 & & & 1 & & 1 \\
\hline Desmochitina minor & 8 & 2 & 45 & 17 & & & 4 & & 3 & 3 \\
\hline Desmochitina erinacea & 6 & & & & & & & & & \\
\hline Eisenackitina sp. 1 & 2 & & & 4 & & & & & & \\
\hline Euconochitina cf. conulus & 5 & & & 1 & & & & & & \\
\hline Lagenochitina capax & 3 & & & & & & & & & \\
\hline Lagenochitina spp. & 6 & & & 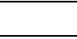 & & & & & & \\
\hline Rhabdochitina magna & $8 ?$ & & 4 & 3 & & & & & & \\
\hline Rhabdochitina spp. & 2 & 1 & 1 & 3 & & & & 1 & & \\
\hline Siphonochitina formosa & 9 & 1 & 7 & 3 & $2 ?$ & & & & & \\
\hline Siphonochitina robusta & 4 & & 2 & $2 ?$ & & & & & & \\
\hline Siphonochitina spp. & 4 & & & 2 & 4 & & & & & \\
\hline Spinachitina multiradiata & 137 & 44 & & $1 ?$ & & & & & & \\
\hline Spinachitina ?cervicornis & 2 & & & & & & & & & \\
\hline Belonechitina micracantha & & $1 ?$ & 7 & 10 & & & & & & \\
\hline Spinachitina spp. & & 2 & 1 & & 2 & 2 & 3 & & & \\
\hline Acanthochitina pudica \# & & & 6 & 13 & & & & & & \\
\hline Belonechitina vulgaris & & & $1 ?$ & & & & & & & \\
\hline Belonechitina sp. 2 & & & 2 & & & & & & & \\
\hline Conochitina parviventer & & & 6 & 7 & & & & & & \\
\hline Cyathochitina campanulaeformis Ikuckersiana group & & & 14 & & & & & & & \\
\hline Desmochitina ovulum & & & 2 & 2 & & & & & & \\
\hline Rhabdochitina usitata & & & 5 & & & & & & & \\
\hline Desmochitina aff. bulla & & & & 2 & & & & & & \\
\hline Kalochitina multispinata & & & & $1 \mathrm{cf}$. & 1 & & & & & \\
\hline Siphonochitina cf. pellucida (sensu Jenkins, 1967) & & & & 1 & & & & & & \\
\hline Belonechitina wesenbergensis & & & & & 35 & 17 & & & & \\
\hline Belonechitina spp. & & & & & 3 & & & 1 & & 1 \\
\hline Eisenackitina spp. & & & & & 3 & & & & & \\
\hline Spinachitina cenvicornis & & & & & 9 & 22 & & & & \\
\hline Angochitina communis & & & & & & & $1+1 ?$ & 12 & 26 & 100 \\
\hline Cyathochitina latipatagium & & & & & & & 1 & 110 & 28 & 90 \\
\hline Hercochitina spp. & & & & & & & $\mathrm{P}$ & & & \\
\hline Fungochitina actonica & & & & & & & 95 & & & \\
\hline Spinachitina katherinae & & & & & & & 257 & & & \\
\hline Acanthochitina latebrosa & & & & & & & & 35 & 12 & 35 \\
\hline Ancyrochitina onniensis & & & & & & & & 40 & 20 & 85 \\
\hline Ancyrochitina spp. & & & & & & & & 1 & 1 & 10 \\
\hline Hercochitina frangiata & & & & & & & & 92 & 11 & 98 \\
\hline Lagenochitina prussica & & & & & & & & & $\mathrm{V}(1)$ & \\
\hline Cyathochitina jenkinsi & & & & & & & & & 7 & 1 \\
\hline Belonechitina capitata / Conochitina elegans group & & & & & & & & & 17 & \\
\hline Ancyrochitina ?spongiosa & & & & & & & & & & $\mathrm{V}(1)$ \\
\hline scolecodonts & $x$ & & & $x$ & $\mathrm{x}$ & $x$ & $x$ & $x$ & $\mathrm{x}$ & $x$ \\
\hline acritarchs & $x$ & & $\mathrm{x}$ & & & & $\mathrm{x}$ & & & \\
\hline total amount of chitinozoans & 354 & 85 & 244 & 172 & 84 & 46 & 416 & 372 & 169 & 560 \\
\hline amount of dissolved rock $(\mathrm{g})$ & 10.68 & 12.15 & 10.41 & 11.30 & $?$ & 15.05 & $?$ & $?$ & $?$ & 10.03 \\
\hline percentage of the residue picked & 50 & 100 & 100 & 100 & $?$ & 100 & $?$ & $?$ & $?$ & 3 \\
\hline
\end{tabular}

Figure 11. Numerical results of this chitinozoan study in the Onny Valley (excluding data from A. Ancilletta, unpub. DEA thesis, Univ. Liège, 1997, and from Jenkins, 1967). '?' in the lower rows refers to the lack of accurate data on the concentration of chitinozoans in the stored residues of Ancilletta. ' $V$ ' stands for a species observed in the part of the residue that was not used for counting or statistical analysis (that is, the part not included in 'percentage of residue picked'). 


\begin{tabular}{|c|c|c|c|c|c|c|c|c|c|c|c|c|c|c|c|c|c|c|c|c|c|c|c|}
\hline \multirow{2}{*}{\begin{tabular}{|c|} 
chitinozoans \\
from the type \\
Caradoc area \\
(South \\
Shropshire): \\
integrated results
\end{tabular}} & \multirow{2}{*}{\begin{tabular}{|c|} 
corresponding levels \\
Jenkins (1967) \\
\\
samples treated in \\
$2004-6($ boldd) $\&$ \\
in 1997 (italic* $)$ \\
\end{tabular}} & \multirow{2}{*}{$\underbrace{\mathrm{c16}}$} & \multicolumn{3}{|c|}{ c13 } & \multirow{2}{*}{ 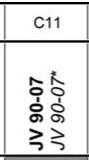 } & \multicolumn{3}{|c|}{ 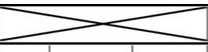 } & \multirow{2}{*}{ 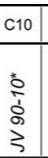 } & \multicolumn{4}{|c|}{ c9 } & \multicolumn{3}{|c|}{$\mathrm{C} 14$} & \multirow{2}{*}{ 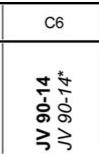 } & \multicolumn{4}{|c|}{$\mathrm{C1}$} & \multirow{2}{*}{ 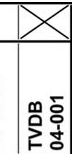 } \\
\hline & & & 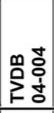 & $\begin{array}{l}\frac{1}{6} \\
\frac{1}{5} \\
\vdots \\
⿱ 亠 䒑 \\
\end{array}$ & 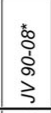 & & $\begin{array}{l}\text { to } \\
\grave{1} \\
\vdots \\
\vdots \\
\end{array}$ & 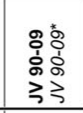 & 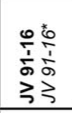 & & 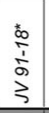 & 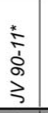 & \begin{tabular}{l}
\multirow{2}{*}{} \\
$\frac{1}{1}$ \\
$\vdots$ \\
3
\end{tabular} & $\begin{array}{l}\frac{0}{0} \\
\frac{1}{1} \\
\vdots \\
\vdots \\
⿱ 亠 䒑\end{array}$ & 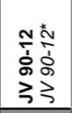 & 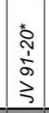 & 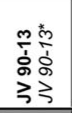 & & 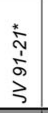 & 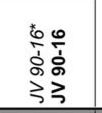 & 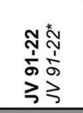 & 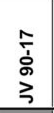 & \\
\hline \multicolumn{2}{|c|}{$\begin{array}{l}\text { B. capitata / C. elegans group } \\
\text { Lagenonchitina baltica }\end{array}$} & & & $3^{*}$ & $1^{*}$ & & & $1^{*}$ & $1^{*}$ & & & $3^{*}$ & & $2^{*}$ & & & & $2^{*}$ & & $1 \% \mathrm{~V}(6)$ & $17-13^{*}$ & - & \\
\hline \multicolumn{2}{|c|}{$\begin{array}{l}\text { Lagenocchitina baltica } \\
\text { Belonechitina brittanica }\end{array}$} & & 8 & & & & & & & & & & & & 1 & & & & & $1^{*}$ & $1^{*}$ & & \\
\hline \multirow{2}{*}{\multicolumn{2}{|c|}{$\begin{array}{l}\text { Belonechititana robusta } \\
\text { Conochitina chydaea }\end{array}$}} & & $9 ?$ & $15^{*}$ & $7^{*}$ & $2 ?$ & & $9 \cdot 8^{*}$ & $8 \cdot 6^{*}$ & & & & & & $1 ? \cdot 1^{*}$ & & 5 & $7^{*}$ & $1^{*}$ & $V(9)$ & $1^{*}$ & & \\
\hline & & & 8 & $4^{*}$ & $5^{*}$ & 1 & & $20-6^{*}$ & $4^{*}$ & & $1^{*}$ & & & $1^{*}$ & $6.5^{*}$ & & & $1^{*}$ & & $1^{*}$ & $1^{*}$ & 2 & \\
\hline Conochitina minnesote & & & 11 & & $5^{*}$ & 1 & & $4 \cdot 7^{*}$ & $4 \cdot 4^{*}$ & & & & & & 1 & & & & & & & 1 & \\
\hline Conochitina Ptigrina & & & $\frac{1}{3}$ & - & & & & & & & & & & & & & & & & & & & \\
\hline$\frac{\text { Conocothitina sp. } 2}{\text { Cyyathochitina campan }}$ & nulaeformis & & $\frac{3}{6}$ & - & & 1 & & 32 & $\frac{2}{51}$ & & & & & & 1 & & & & & $\mathrm{~V}(\mathbf{2})$ & & 1 & \\
\hline Cyathochitina cf. calli & & & 9 & & & & & 2 & 4 & & & & & & & & & & & & & & \\
\hline Cyathochitina kuckers & & & $\frac{1}{2}$ & $8^{*}$ & $8^{*}$ & $15-8^{*}$ & $4^{*}$ & $31 \cdot 104^{*}$ & $6.65^{*}$ & $1^{*}$ & $1^{*}$ & $6^{*}$ & & 9 & $2^{*}$ & & $1^{\circ}$ & $17-10^{\circ}$ & $20^{*}$ & $25^{5} \mathrm{~V}(1)$ & $41-30^{\circ}$ & 55 & 97 \\
\hline $\begin{array}{l}\text { Cyathochitina sp. } 1 \\
\text { Desmochitin minor }\end{array}$ & & & $\frac{2}{8}$ & & & 2 & & 45 & 17 & & & & & & & & & 4 & & $\frac{1 / 31}{V(3)}$ & 3 & & 3 \\
\hline Desmochitina erinace & & & 6 & & & & & 45 & & & & & & & & & & 4 & & $v(0)$ & 0 & & \\
\hline Eisenackitina sp. 1 & & & 2 & & & & & & 4 & & & & & & & & & & & & & & \\
\hline Euconochitina cf. con & & & 5 & & & & & & 1 & & & & & & & & & & & & & & \\
\hline Lagenochitina capax & & & 3 & & & & & & & & & & & & & & & & & & & & \\
\hline Rhabdochitina magna & & & $8 ?$ & $1^{*}$ & $5^{*}$ & & $2^{*}$ & $4 \cdot 6^{*}$ & 3 & & & & & & & & & $2^{\star}$ & $7^{*}$ & $5^{*}$ & $2^{\star}$ & & \\
\hline Siphonochitina formos & & & 9 & & & 1 & $5^{*}$ & $7 \cdot 7^{*}$ & $3-3^{*}$ & & & & & & $2 ?$ & & & & & & & & \\
\hline Siphonochitina robuste & & & 4 & & & & $1^{*}$ & $2 \cdot 10^{*}$ & $2 ? \cdot 3^{*}$ & & & & & & & & & & & & & & \\
\hline Spinachitina multiradia & & & 137 & $48^{*}$ & $14^{*}$ & $44-29^{*}$ & $1 * ?$ & $7 * ?$ & $? 1-4^{*}$ & & & & & $4^{*} ?$ & & & & $6^{*} ?$ & & & & & \\
\hline Spinachitin & & & 2 & & & & & & & & & & & & & & & & & & & & \\
\hline Belonechitina micraca & & & & & & 1? & & 7 & 10 & & & & & & & & & & & & & & \\
\hline Spinachitina spp. & & & & & & 2 & & 1 & & & & & & & 2 & & 2 & 3 & & $V(1)$ & & & \\
\hline Siphonochitina clavate & & & & & & & & & & & & & & & & & & & & & & & \\
\hline $\begin{array}{l}\text { Lagenochitina shelven } \\
\text { Conochitina lepida }\end{array}$ & & & & - & - & & & 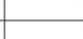 & & & & & & & & & & & & & & & \\
\hline 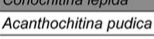 & & & & & & & & $6.5^{*}$ & $13 \cdot 17^{*}$ & & & & & & & & & & & & & & \\
\hline Belonechitina vulgaris & & & & & & & & 1? & & & & & & & & & & & & & & & \\
\hline Belonechitina sp. 2 & & & & & & & & 2 & & & & & & & & & & & & & & & \\
\hline Conochitina parvivente & & & & & $1^{*}$ & & & $6 \cdot 10^{*}$ & $7 \cdot 3^{*}$ & & & & & & $1^{*}$ & & & & & & & & \\
\hline $\begin{array}{l}\text { C. campanulaeformis } \\
\text { Desmochitina ovulum }\end{array}$ & kuckersiana group & & & & & & & 14 & $2.2 f^{*}$ & & & & & $3 c * t$ & & & & & & & & & \\
\hline $\begin{array}{l}\text { Desmochitina avulum } \\
\text { Rhabdochitina usitata }\end{array}$ & & & & & & & & 2 & $2 \cdot 2 c^{2 f^{*}}$ & & & & & & & & & & & & & & \\
\hline Desmochitina aff. bull & & & & & & & & & 2 & & & & & & & & & & & & & & \\
\hline Kalochitina multispinat & & & & & & & & & 1 cf. & & & & & & 1 & & & & & & & & \\
\hline Siphonochitina cf. pel & Ellucida & & & & & & & & 1 & & & & & & $250^{4}$ & & & & & & & & \\
\hline $\begin{array}{l}\text { Belonechitina wesenb } \\
\text { Spinachitina cervicom }\end{array}$ & \begin{tabular}{l|} 
bergensis \\
nis (former alaticomis)
\end{tabular} & & & & & & & & & & & & & & $\begin{array}{l}\frac{35 \cdot-40^{*}}{9-P<2^{*}} \\
\end{array}$ & & $\frac{17 \cdot 1^{*}}{22-P 8^{*}}$ & $P ?^{* *}$ & & & & & \\
\hline Angochitina communis & & & & & & & & & & & & & & & & & & $1+1 ?$ & $10^{\circ}$ & $9^{*} / \mathrm{V}(13)$ & $26-3^{*}$ & 12 & 100 \\
\hline Cyathochitina latipatas & & & & & & & & & & & & & & & & & & 1 & & $V(4)$ & 28 & 110 & 90 \\
\hline Fungochitina actonica & & & & & & & & & & & & & & $18^{*}$ & & & & $95-10^{*}$ & $1^{*}$ & & & & \\
\hline Spinachitina katherina & & & & & & & & & & & & & & & $P<12^{*}$ & & $P<8^{*}$ & $257-P<104^{*}$ & & & & & \\
\hline Hoegisphaera co & & & & & & & & & & & & & & & & & & & & & & & \\
\hline Acanthochitina latebro & barbata ) & & & & & & & & & & & & & $1^{*}$ & & & & & $22^{*}$ & $20^{\circ} \mathrm{V}(13)$ & $12-43^{\circ}$ & 35 & $\begin{array}{l}35 \\
85\end{array}$ \\
\hline $\begin{array}{l}\text { Ancyrochitina onniens } \\
\text { Hercochitina frangiata }\end{array}$ & & & & & & & & & & & & & & & & & $2^{*}$ & & $35^{*}$ & $\frac{10 \% \mathbf{V}(\mathbf{9})}{30 \%}$ & $\frac{20-1^{*}}{11 \cdot 1^{*}}$ & $\begin{array}{l}40 \\
92\end{array}$ & $\begin{array}{l}85 \\
98 \\
\end{array}$ \\
\hline Ancyrochitina ?spong & & & & & & & & & & & & & & & & & & & & & & & $v(1)$ \\
\hline $\begin{array}{l}\text { Cyathochitina off jenk } \\
\text { Lagenochitina ar ussicsick }\end{array}$ & & & & & & & & & & & & & & & & & & & $19^{*}$ & $10^{*} \mathrm{~V}(48)$ & $7-28^{*}$ & & 1 \\
\hline Forma & ations: & 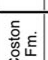 & & $\begin{array}{l}\text { eathen W } \\
\text { ermatior }\end{array}$ & $\begin{array}{l}\text { Nood } \\
\text { on }\end{array}$ & $\begin{array}{l}\text { Glen- } \\
\text { burrell } \mathrm{Fm} .\end{array}$ & & Horderley Sa & ndstone $\mathrm{Fr}$ & & Altern & ata Li & nesto & & Cheney & giv & ville Fm. & $\begin{array}{l}\text { Acton Scott } \\
\text { Fm. }\end{array}$ & & & Formation & & \\
\hline
\end{tabular}

Figure 12. Overview of the combined data obtained from the three chitinozoan studies on the southern Caradoc area by Jenkins (1967: shaded), Ancilletta (unpub. DEA thesis, Univ. Liège, 1997: number of specimens in italic, with '*') and ourselves (number of specimens in bold typeface). Selected samples from Jenkins (1967, table I, p. 482) are also included, which are not from the Onny Valley section itself; for example, C16 is from as far away as the northern (Chatwall) tract of the Caradoc Inlier. ' $\mathrm{P}$ ' stands for species present but lacking absolute abundance data in the work of Ancilletta (unpub. DEA thesis, Univ. Liège, 1997); 'V' stands for a species observed in a sample of residue studied in 2005, but in the part of the residue that was not used for counting or statistical analysis ('percentage of residue picked' in Fig. 11).

(5) One of the authors (TVDB) was able to examine the vast Canadian chitinozoan collections of A. Achab and E. Asselin at the INRS-ETE in Quebec. Following re-study of the Onny Valley material as well as material from Pointe Laframboise (Anticosti Island, Quebec: Achab, 1981), we reject Ancilletta's synonymy (unpub. DEA thesis, Univ. Liège, 1997) of the Hirnantian Spinachitina taugourdeaui and his ornamented Spinachitina alaticornis. The latter species is called Spinachitina katherinae in the presentaccount.

(6) Likewise, the recognition of Hercochitina gamachiana in the Onny Valley section by Ancilletta (unpub. DEA thesis, Univ. Liège, 1997) proved incorrect after comparison with the original Quebec material.

(7) After study of Acanthochitina barbata from Anticosti Island (Achab, 1977; our own observations in Quebec, 2004) and from Estonia (our own SEM observations on material kindly provided by Jaak Nõlvak), we concluded that the specimens from Onny Valley, originally attributed to A barbata by both Jenkins (1967) and Ancilletta (unpub. DEA thesis, Univ. Liège, 1997), are in fact a different species, Acanthochitina latebrosa (Vandenbroucke, 2008b).

(8) The systematic problems with Angochitina communis, its suggested synonymy with Belonechitina hirsuta, and the resulting stratigraphical implications are commented on by Vandenbroucke (2008b). We followed the Jenkins holotype definition, adding forms with shorter and aligned spines to the species, which were called Belonechitina sp. B by Ancilletta (unpub. DEA thesis, Univ. Liège, 1997). 


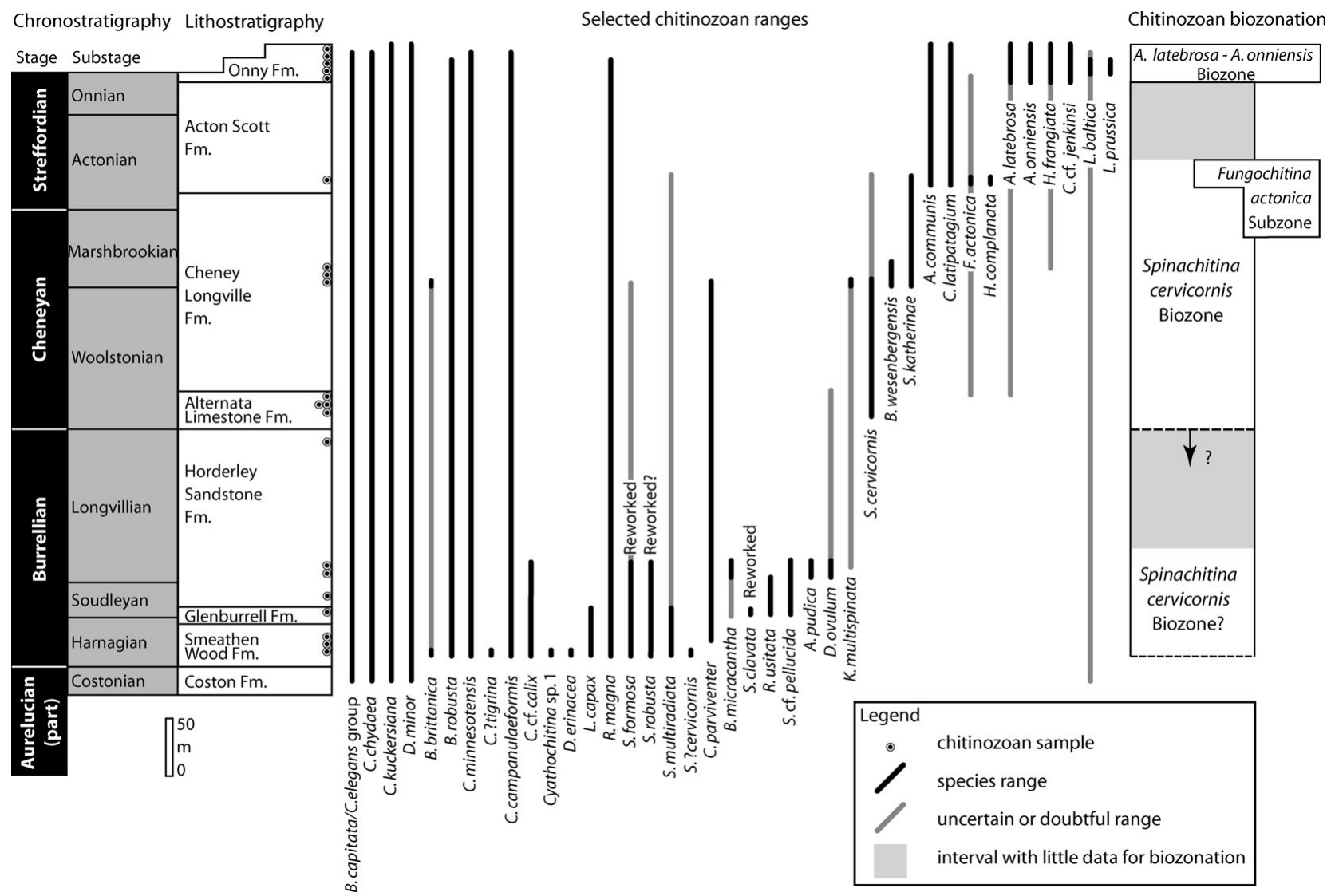

Figure 13. Range chart and a biozonation in the Onny Valley section (southern Caradoc area), integrating the three chitinozoans studies in the area (Jenkins, 1967; A. Ancilletta, unpub. DEA thesis, Univ. Liège, 1997; this study). The grey lines represent uncertain species ranges (e.g. for end-range specimens only found in one of the three studies, in low numbers, such as the doubtful occurrences of $L$. baltica low in the stratigraphy, see text Section 4 and Fig. 12). The base of the Spinachitina cervicornis Biozone is ill-constrained because of minor systematic problems. The left-hand side columns are after Rushton et al. (2000).

\section{Interpretation, stratigraphical value and biozonation}

\section{5.a. Shelve Inlier}

Although the samples yielded a high number of moderately to well-preserved chitinozoans, the assemblage displayed a rather low diversity and was virtually devoid of stratigraphically important species.

Superficially looking at the Lower Wood Brook range chart (Fig. 6), one might have the impression that a large number of new species appear immediately below the base of the Nemagraptus gracilis Biozone. This is, however, not the case, the visual effect being caused by the unusually small scale of the figure, necessary to include the two lowermost samples. The effect is enhanced by the large unsampled interval of more than 200 m between S224 and S211 and the rather poor preservation in the lowermost two samples. The detailed range chart (Fig. 7) is still at a fairly small scale, representing a little less than $30 \mathrm{~m}$ of shales, but it shows that no chitinozoan species has its first or last occurrence around the base of the $N$. gracilis Biozone.

Most of the chitinozoans identified at species level have an extensive range through the thick section, and, based on the literature, through a large part of the Ordovician. Well-known examples are Conochitina chydaea, Belonechitina micracantha, Cyathochitina cf. calix, Cyathochitina campanulaeformis, Cyathochitina campanulaeformis-kuckersiana group, Desmochitina ovulum, Desmochitina minor, etc.

Species having more stratigraphical potential are: Eisenackitina ?rhenana, Eisenackitina inconspicua and Linochitina aff. pissotensis in the Lower Wood Brook section; Conochitina tigrina, Spinachitina bulmani and Siphonochitina robusta in the Spy Wood Brook section (although the latter species may be reworked). These species, however, occur in much lower numbers than the first group.

\section{5.a.1. Eisenackitina rhenana Subzone?}

Eisenackitina ?rhenana has also been reported from the Swedish Fågelsång section, GSSP (Global Stratotype Section and Point) for the base of the Upper Ordovician; the chitinozoans from this section have been studied by Bergström et al. (2000) and Vandenbroucke (2004). E. ?rhenana is slightly larger than Eisenackitina rhenana and lacks a clearly developed flexure (Vandenbroucke, 2004). It has a longer range than E. rhenana, the subzonal index fossil used as a proxy for the base of the Upper Ordovician and it extended both below and above the lowest and highest records of E. rhenana 

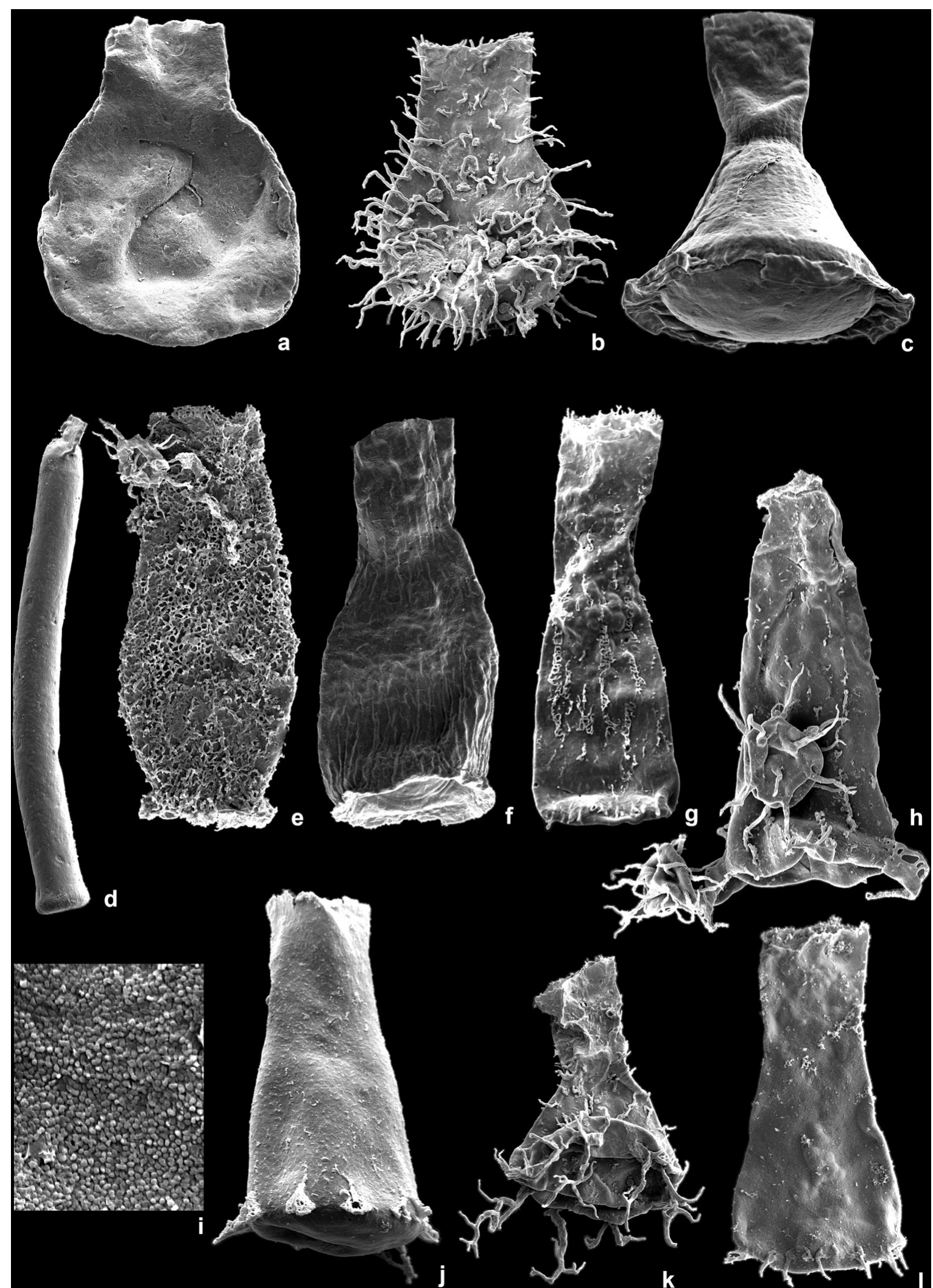

Figure 14. Chitinozoans from the Onny Valley section. All measurements in micrometres $(\mathrm{L} \times \mathrm{Dp}$, or $\mathrm{L} \times \mathrm{Dp} \times \mathrm{Dc}$, or $\mathrm{L} \times \mathrm{Dp} \times \mathrm{Dc} \times$ Lc). For abbreviations, see Paris (1981): L - total length, Dp - chamber diameter, Dc - diameter of oral tube, Lc - length of oral tube. (a) Lagenochitina prussica, sample 90-16 $(190 \times 160 \times 70)$; (b) Angochitina communis, sample 91-22 $(100 \times 65 \times 38)$; (c) Cyathochitina latipatagium, sample 90-16 $(210 \times 150 \times 55)$; (d) Belonechitina capitata-Conochitina elegans group, sample $91-22(630 \times 65 \times$ 50); (e) Acanthochitina latebrosa (with attached acritarch), sample 90-16 (350 × $140 \times 100)$; (f) Cyathochitina cf. jenkinsi, sample 04-001 $(260 \times 115 \times 65) ;(\mathrm{g})$ Hercochitina frangiata, sample 90-16 $(220 \times 80 \times 45)$; (h) Spinachitina katherinae (with attached acritarchs), sample 90-14 $(200 \times 90 \times 35)$; (i) detail of the granular ornamentation of L. prussica; see (a); (j) Spinachitina cervicornis, sample 90-13 $(130 \times 60 \times 35)$; (k) Ancyrochitina onniensis, sample 91-22 $(100 \times 65 \times 25)$; (1) Spinachitina multiradiata, sample TVDB 04-004 $(140 \times 60 \times 40)$. 
(Vandenbroucke, 2004). However, the difference between the ranges of both species in Fågelsång is only in the order of magnitude of a couple of metres. In addition, a species listed by Nõlvak \& Grahn (1993) as being characteristic for the upper part of the Laufeldochitina stentor Biozone and its E. rhenana Subzone, namely Conochitina tigrina, has been discovered in the Spy Wood Brook section in the same sample as the topmost find of E. ?rhenana. According to Nõlvak (2005), C. tigrina has been found in the upper part of the E. rhenana Subzone in the Mehikoorma core. Nõlvak (2001) also shows the presence of $C$. tigrina in the topmost part of the $L$. stentor Biozone of the Valga (10) core, immediately above the E. rhenana Subzone. In short, we consider their coexistence to be quite stable. The absence of the index fossil from the Shelve area, or its imperfect preservation hampering positive identification, does not allow us to recognize the $E$. rhenana Subzone of the L. stentor Biozone as such, but we will provisionally use the E. rhenana Subzone? to indicate proximity to the level at Fågelsång. We have not found E. ?rhenana or any other species indicative of the E. rhenana Subzone? in the upper half of the Rorrington Shale Formation in the sections studied, which explains the apparent gap between finds of the subzone in its different localities on Figures 6 and 8.

Eisenackitina inconspicua has been defined in the Fågelsång section (Vandenbroucke, 2004), where unfortunately it is one of the longer-ranging species, and it is difficult to evaluate its stratigraphical range. The stratigraphical value of the species has yet to be confirmed, although its presence in the same graptolite biozones in both Fågelsång and the Shelve areas is an important indication of its stratigraphical potential.

Linochitina pissotensis is the index fossil of the eponymous northern Gondwana biozone defined as the total range zone of L. pissotensis (Paris, 1990). In contrast to our own previous findings (Vandenbroucke et al. 2003), the specimens are not identical to the ones recovered from Gondwana (Paris, pers. comm. 2003, 2005 ) and are kept in open nomenclature. The biozone could therefore not be identified in Lower Wood Brook.

Al-Hajri (1995) also reported a remarkable similarity between the Saudi Arabian faunas and the Shropshire fauna in Jenkins (1967). However, he recorded Laufeldochitina robusta (a synonym for Siphonochitina robusta) from much lower levels than recorded in our study from the Shelve area: lower Llanvirn in Saudi Arabia, in contrast to Caradoc-Burrellian in the Onny Valley (see next Section).

Conochitina tigrina, Siphonochitina robusta and (?) Spinachitina bulmani allow us to link the Spy Wood Brook section with the type Caradoc area, as discussed in the next two paragraphs.

\section{5.b. Onny Valley}

Typical Llanvirn forms have been noticed in the section, such as Siphonochitina formosa and Siphonochitina clavata, and they are thought to have been reworked (Fig. 13), not least because of earlier reports of reworking of acritarchs in the section (Turner, 1982), and because of their much shorter range on other palaeocontinents, where they are used for biostratigraphical purposes. The presence of other species that are known from Llanvirn times onwards, such as Siphonochitina robusta and Conochitina parviventer, is less easily explained by reworking, as they have also been observed in the contemporaneous Caradoc Shelve Inlier deposits. The latter were formed in deeper water settings, where reworking is less probable, at least by the mechanism described by Turner (1982). Unlike the case with acritarchs, no Tremadocian chitinozoans were found, easily enough explained considering the early stage of chitinozoan dispersal during the Tremadocian.

The lowermost levels from the section yield a fauna described by Jenkins (1967) as 'Assemblage one' and comparable to the rather uniform chitinozoan assemblage recovered from the Shelve area. Accurate correlations are difficult, however, due to the rather long stratigraphical range of most species. Conochitina tigrina might prove interesting to link both sections, although only one, doubtfully identified, specimen has been reported from the Onny Valley. In addition, Spinachitina bulmani, found in levels higher in the Aldress Shale Formation in Spy Wood Brook, is morphologically very close to Spinachitina multiradiata from the Smeathen Wood Formation, although no certainty exists about the true FAD of the species in both sections.

Spinachitina multiradiata is an interesting species. As already mentioned, the basal spines remind us of the slightly more complex appendices of Spinachitina cervicornis. The latter species bears ornamentation on the chamber wall, while $S$. multiradiata is smooth. However, Nõlvak \& Grahn (1993, plate III, a, p. 256), in the paper in which they erect the $S$. cervicornis Biozone, figure a smooth $S$. cervicornis specimen; it is morphologically very close to $S$. multiradiata found in the Onny Valley.

Based on the succession in the Onny Valley, we propose an evolutionary lineage of progressively more complex ornamentation within the genus Spinachitina. It starts with Spinachitina multiradiata, a form with large basal spines already a bit further developed than in the shorter-spined Spinachitina bulmani (Jansonius, 1964). Higher up, Spinachitina cervicornis bears more complex, comb-like appendices, but is otherwise smooth to lightly ornamented; Spinachitina katherinae has similar appendices, but bears ornamentation on the chamber wall, consisting of crests of membranes or arches formed by spines with connected tops, aligned parallel with the vesicle's longitudinal axis and continuing on the appendices (see Vandenbroucke, 2008b, text-fig. 8).

It is at present unclear where the base of the Spinachitina cervicornis Biozone ought to be drawn exactly. In Baltica, Spinachitina multiradiata straddles the base of the Spinachitina cervicornis Biozone 
and at the base of the Onny Valley section, a few specimens of Spinachitina ?cervicornis have been observed (Fig. 13). In the Onny Valley, the lowest unambiguous Spinachitina cervicornis are from the Alternata Limestone Formation (following our colleagues in Baltica, where S. alaticornis is considered a junior synonym of $S$. cervicornis: Yngve Grahn, pers. comm. 2006; Jaak Nõlvak, pers. comm. 2007). For the time being, we attribute the interval below the Alternata Limestone Formation only tentatively to the $S$. cervicornis Biozone. If eventually it becomes clear that our $S$. multiradiata or S. ?cervicornis specimens are indeed identical to the Baltoscandic $S$. cervicornis zonal index fossils, then the base of the $S$. cervicornis Biozone can be lowered to the Costonian. It is worthwhile to note that in Laurentian sections, the evolutionary lineage is not seen; there, the only suggested evolution within S. bulmani is the increasing slenderness of its vesicle (Vandenbroucke, Verniers \& Clarkson, 2003).

\section{5.c. Onny Valley biozonation}

In summary, the following biozones can be observed in the Onny Valley section, from bottom to top (Fig. 13).

\section{5.c.1. Spinachitina cervicornis Biozone}

The biozone was defined by Nõlvak \& Grahn (1993) in Baltoscandia as corresponding to the total range of the index fossil, a definition that is emended here so the zone ranges up to the lowest occurrence of the index species of the overlying biozone; we also take into account that these authors consider $S$. cervicornis and $S$. alaticornis to be synonymous (Grahn, pers. comm.; Nõlvak, pers. comm.). In the Onny Valley section, the biozone can thus easily be recognized in the Alternata Limestone, Cheney Longville and Acton Scott formations, corresponding to Cheneyan to midStreffordian age. Lower down in the stratigraphy, in the Smeathen Wood, Glenburrell and Horderley Sandstone formations (Burrellian), the biozone has only been tentatively recognized, by the presence of Spinachitina multiradiata and Spinachitina ?cervicornis. Desmochitina juglandiformis, known from this zone in Baltoscandia and present in other sections in the UK, is absent in south Shropshire. Spinachitina katherinae (remarkably similar to the index fossil), Belonechitina wesenbergensis and Acanthochitina pudica are easily recognizable, accessory species within this biozone, although the latter ranges within the lower part of the biozone that is only doubtfully attributed to it.

\section{5.c.2. Fungochitina actonica Subzone}

The subzone is defined by the first occurrence of Fungochitina actonica up to the lowest occurrence of the index species of the overlying biozone. In the Onny Valley the species is typically recovered from the Acton Scott Formation (Actonian). The records of the index species from the Alternata Limestone (A. Ancilletta, unpub. DEA thesis, Univ. Liège, 1997; see Fig. 13) are unconfirmed.

\section{5.c.3. Acanthochitina latebrosa-Ancyrochitina onniensis Biozone}

The biozone is defined by the first occurrence of Acanthochitina latebrosa up to the lowest occurrence of the index species of the overlying biozone, excluding the single specimen from the Alternata Limestone Formation reported by Ancilletta (unpub. DEA thesis, Univ. Liège, 1997). In the Onny Valley, the biozone is restricted to the Onny Formation (Onnian). The accessory index fossil Ancyrochitina onniensis has the same range as the zonal index fossil. Both are joined by Hercochitina frangiata, Cyathochitina cf. jenkinsi, Angochitina communis, rare Lagenochitina prussica and, probably, Lagenochitina baltica. Angochitina communis has been excluded from the zonal definition, because of taxonomic problems (Vandenbroucke, 2008b).

The three biozones correspond well with "Assemblages two, three and four' as reported by Jenkins (1967).

Inter-section correlations are discussed at length in a paper by Vandenbroucke (2008a), but the most important stratigraphical links are listed below. Despite the problems concerning the systematics of Angochitina communis, the species does allow correlation with the Cross Fell Inlier (northern England). There, at lower stratigraphical levels, the species has been used for definition of a local biozone, as no other usable chitinozoans were available (Vandenbroucke, Rickards \& Verniers, 2005), this in contrast with the practice in Onny Valley, where species with fewer systematic problems were preferentially used for biozone definition. Specimens of $A$. communis from both sections, illustrated in plates 12 and 22.2 of Vandenbroucke (2008b), show their identical appearance. Acanthochitina latebrosa has also been reported from the Fungochitina spinifera Zone in Whitland in south central Wales (Vandenbroucke et al. 2008); likewise, Lagenochitina prussica and Lagenochitina baltica, normally typical of the $F$. spinifera Zone and younger, have been reported from the Onny Valley. These records represent very low yields of specimens (especially when compared to the remainder of the assemblages), which obscures their potential to correlate these two biozones.

Apart from the minor taxonomic problems, the recognition of the Spinachitina cervicornis Biozone allows straightforward correlation with the upper Haljala, Keila and lower Oandu stages in Baltoscandia (Nõlvak \& Grahn, 1993; Webby et al. 2004; Nõlvak, Hints \& Männik, 2006). Additionally, the Scottish Hartfell Score section, formerly proposed as a GSSP for the base of the Katian stage of the international Upper Ordovician Series, contains chitinozoans from the S. cervicornis Biozone (Zalasiewicz and 
others, unpub. data, 2004: http://www.ordovician.cn). Interestingly, a section supplemental to the selected Black Knob Ridge GSSP for the base of the Katian stage, known as 'section D', yields well-preserved chitinozoans in two levels, one below and one above the base of the Katian. The lowest of these levels was attributed to the $S$. cervicornis Biozone; the higher one remained unzoned but was dated to the Baltoscandian Keila stage (Goldman and others, unpub. data, 2005: http://www.ordovician.cn). Possible ties with the upper part of the sections studied from the Shelve area have already been mentioned, although the assemblage used consists of rather long-ranging species, with the exception of Conochitina tigrina, but the stratigraphical value of this species has to be treated with caution, as only a single, questionably identified, specimen was found in the Onny Valley.

This paper is a contribution to an ongoing construction of an Upper Ordovician chitinozoan biozonation in the UK, tied to British chronostratigraphy in its original type areas (Vandenbroucke, 2008a).

\section{Conclusions}

With a few exceptions, the assemblage recovered in the Shelve Inlier consists mainly of long-ranging species. Although a large number of chitinozoans were studied, little specific variation occurs throughout the $400 \mathrm{~m}$ thick succession, and no particular faunal change in the chitinozoan assemblage was observed at or near the base of the Nemagraptus gracilis Biozone. The chitinozoans allow us to conclude only that this level is stratigraphically close to the base of the Upper Ordovician, although a good, unambiguous proxy for this boundary has not been recognized, as has been in the GSSP for that level, at Fågelsång. A provisional, local Eisenackitina rhenana Subzone? is proposed, using the range of the species retained under open nomenclature, and the presence of Conochitina tigrina in the topmost part of the biozone. The presence of the formerly recognized upper part of the northern Gondwana Linochitina pissotensis Biozone across the Llanvirn-Caradoc boundary (Vandenbroucke et al. 2003) is here rejected.

The rich and well-preserved chitinozoan fauna of Caradoc type area, along the Onny River in the south Shropshire region, has been re-evaluated to attribute the assemblages to more generally applicable biozones. Interestingly, almost the entire section can be interpreted as belonging to the originally Baltoscandian Spinachitina cervicornis Biozone; in the Cheneyan and lower to middle Streffordian parts of the section this biozone is certainly present, but in the lower (Burrellian) part, the attribution is rather doubtful due to a systematic problem concerning Spinachitina ?cervicornis and Spinachitina multiradiata. The top part of the section, namely the Onny Formation, has been attributed to a (local) Acanthochitina latebrosaAncyrochitina onniensis Biozone. An accessory species of this zone is also present in the uppermost
Caradoc beds in the Cross Fell Inlier and the biozone has some poorly represented species in common with the $F$. spinifera Zone in Whitland. The previously established presence of Acanthochitina barbata in the Onnian is rejected. On the whole, the chitinozoan fauna from the Onny Valley is rather different from the faunas from other sections in the Anglo-Welsh Basin, a separation not easily explained, unless perhaps by different palaeo-environmental settings.

Acknowledgements. Florentin Paris and one anonymous reviewer provided helpful and constructive comments. We thank Richard Bettley (Oxford University) for providing samples, Derek Siveter (Oxford University), Brian Chatterton (University of Alberta) and Geert Van Grootel (Ghent University) for assistance in the field, Jaak Nõlvak (Tallinn University of Technology) for providing specimens, Jan Vanmeirhaeghe (Ghent University), Florentin Paris (University of Rennes I, CNRS), Aicha Achab and Ester Asselin (INRS-ETE, University of Quebec), Yngve Grahn (Petrobras, Brazil) and Jaak Nõlvak for help with identifications, Charles Wellman, Ken Dorning and Duncan Mclean for arranging access to the Jenkins collection at Sheffield University, Sabine Vancauwenberghe for the laboratory preparations, and Stephen Louwye, Achilles Gautier (Ghent University), and Aicha Achab (INRS-ETE, University of Quebec) for commenting on an earlier version of this paper (as part of TVDB's Ph.D. jury). Although included in the authorship of this paper, in acknowledgement of his effort, we were unable to locate or re-contact Antonio Ancilletta in person. This paper is a contribution to the IGCP 503 project (Ordovician Palaeogeography and Palaeoclimate) and the FWO research project 3G027105. FWO-Flanders is sincerely acknowledged for funding our projects.

\section{References}

ACHAB, A. 1977. Les chitinozoaires de la zone à Dicellograptus complanatus Formation de Vauréal, Ordovicien supérieur, Ile d'Anticosti, Québec. Canadian Journal of Earth Sciences 14, 413-25.

ACHAB, A. 1981. Biostratigraphie par les Chitinozoaires de l'Ordovicien Supérieur-Silurien Inférieur de l'Ile d'Anticosti. Résultats préliminaires. In Field Meeting, Anticosti-Gaspé, Québec. Vol. II: Stratigraphy and Paleontology (ed. P. J. Lespérance), pp. 143-57. Quebec: IUGS Subcommission on Silurian Stratigraphy, Ordovician-Silurian Boundary Working Group.

AL-HAJRI, S. 1995. Biostratigraphy of the Ordovician chitinozoa of northwestern Saudi Arabia. Review of Palaeobotany and Palynology 89, 27-48.

BANCROFT, B. B. 1933. Correlation tables of the stages Costonian-Onnian in England and Wales. Gloucester: Blakeney (privately printed), $4 \mathrm{pp}$.

Bergström, S. M., Finney, S. C., Chen Xu, PÅlsson, C., WANG ZHI-HAO \& GRAHN, Y. 2000. A proposed global boundary stratotype for the base of the Upper Series of the Ordovician System: the Fågelsång section, Scania, southern Sweden. Episodes 23, 102-9.

BetTley, R. M., ForTey, R. A. \& Siveter, D. J. 2001. Highresolution correlation of Anglo-Welsh Middle to Upper Ordovician sequences and its relevance to international chronostratigraphy. Journal of the Geological Society, London 158, 937-52.

CAVE, R. \& Hains, B. A. 2001. Geology of the country around Montgomery and the Ordovician rocks of the 
Shelve area. Memoir for the 1:50.000 Geological Sheet 165 with part of Sheet 151 (Welshpool). London: Her Majesty's Stationery Office.

DEAN, W. T. 1958. The faunal succession in the Caradoc series of south Shropshire. Bulletin of the British Museum (Natural History) Geology 3, 193-231.

DEAN, W. T. 1960. The Ordovician Rocks of the Chatwall District, Shropshire. Geological Magazine 97, 163-71.

DEAN, W. T. 1964. The geology of the Ordovician and adjacent strata in the southern Caradoc District of Shropshire. Bulletin of the British Museum (Natural History) Geology 9, 259-96.

Fortey, R. A., HaRPer, D. A. T., Ingham, J. K., OWEN, A. W., Parkes, M. A., Rushton, A. W. A. \& WoODCOCK, N. H. 2000. A revised correlation of Ordovician Rocks in the British Isles. Geological Society of London, Special Report no. 24, 83 pp.

Fortey, R. A., HARPer, D. A. T., Ingham, J. K., OWEN, A. W. \& Rushton, A. W. A. 1995. A revision of Ordovician series and stages of the historical type area. Geological Magazine 132, 15-30.

Hughes, R. A. 1989. Llandeilo and Caradoc Graptolites of the Builth and Shelve Inliers. Monograph of the Palaeontographical Society no. 141, 89 pp.

HURST, J. M. 1979. The stratigraphy and brachiopods of the upper part of the type Caradoc of south Salop. Bulletin of the British Museum (Natural History) Geology 32, 183-304.

JANSONIUS, J. 1964. Morphology and classification of some Chitinozoa. Bulletin of Canadian Petroleum Geology 12, 901-18.

Jenkins, W. A. M. 1967. Ordovician Chitinozoa from Shropshire. Palaeontology 10, 436-88.

LYNAS, B. D. T. 1985. Geological notes and local details for 110.000 sheet SO 29 NE (Chirbury and Priest Weston). Keyworth, Nottingham: British Geological Survey.

Murchison, R. I. 1839. The Silurian System. London: John Murray.

NõLVAK, J. 2001. Distribution of Chitinozoans. In Estonian Geological Sections, Bulletin 3, Valga (10) drill core (ed. A. Poldvere), pp. 8-10. Geological Journal of the Geological Survey of Estonia, Tallinn.

NÕLVAK, J. 2005. Distribution of Ordovician Chitinozoans. In Estonian Geological Sections, Bulletin 6, Mehikoorma (421) core (ed. A. Poldvere), pp. 20-2. Geological Journal of the Geological Survey of Estonia, Tallinn.

NÕLVAK, J. \& GRAHN, I. 1993. Ordovician chitinozoan zones from Baltoscandia. Review of Palaeobotany and Palynology 79, 245-69.

NÕLVAK, J., HINTS, O. \& MÄNNIK, P. 2006. Ordovician timescale in Estonia: recent developments. Proceedings of the Estonian Academy of Sciences 55(2), 95-108.

PARIS, F. 1981. Les chitinozoaires dans le Paléozoïque du sud-ouest de l'Europe (cadre géologique - étude systématique - biostratigraphie). Mémoire de la Société géologique et minéralogique de Bretagne no. 26, 1-496.

PARIS, F. 1990. The Ordovician chitinozoan biozones of the Northern Gondwana Domain. Review of Palaeobotany and Palynology 66, 181-209.

Pocock, R. W., Whitehead, T. H., Wedd, C. B. \& ROBERTSON, T. 1938. Shrewsbury district, including the Hanwood Coalfield (Sheet 152). Memoir of the Geological Survey of the United Kingdom.

Rushton, A. W. A., Owen, A. W., Owens, R. M. \& PrigmoRe, J. K. 2000. British Cambrian to Ordovician Stratigraphy. Geological Conservation Review Series, no. 18. Peterborough, UK: Joint Nature Conservation Committee, $435 \mathrm{pp}$.
Savage, N. M. \& Bassett, M. G. 1985. Caradoc-Ashgill conodont faunas from Wales and the Welsh Boderland. Palaeontology 28, 679-713.

Smith, N. J. P. \& Rushton, A. W. A. 1993. Cambrian and Ordovician stratigraphy related to structure and seismic profiles in the western part of the English Midlands. Geological Magazine 130, 665-71.

STRACHAN, I. 1986. The Ordovician graptolites of the Shelve District, Shropshire. Bulletin of the British Museum (Natural History) Geology series 40, 1-58.

TURNER, R E. 1982. Reworked acritarchs from the type section of the Ordovician Series, Shropshire. Palaeontology 25, 119-43.

VANDENBROUCKE, T. R. A. 2004. Chitinozoan biostratigraphy of the Upper Ordovician Fågelsång section, Scania, southern Sweden. Review of Palaeobotany and Palynology, 130, 217-38.

VANDENBROUCKE, T. R. A. 2008a. An Upper Ordovician Chitinozoan Biozonation in British Avalonia (England \& Wales). Lethaia 41, 275-94.

VANDENBROUCKE, T. R. A. $2008 b$ (for 2007). Upper Ordovician chitinozoans from the historical type area in the UK. Palaeontographical Society Monograph no. 628, Vol. 161, 113 pp.

Vandenbroucke, T. R. A., Fortey, R. A., Siveter, D. J. \& RICKARDS, R. B. 2003. Chitinozoans from key sections in the Upper Ordovician Series: new GSSP's and classical British sections. In Ordovician from the Andes (eds G. L. Albanesi, M. S. Beresi \& S. H. Peralta), p. 151. Proceedings of the 9th International Symposium on the Ordovician System. INSUGEO, Serie Correlación Geológica 17.

VANDENBROUCKe, T. R. A., Rickards, R. B. \& Verniers, J. 2005. Upper Ordovician Chitinozoan biostratigraphy from the Type Ashgill Area (Cautley district) and the Pus Gill section (Dufton district, Cross Fell Inlier), Cumbria, Northern England. Geological Magazine 142, 783-807.

VAndenbroucke, T. R. A., Verniers, J. \& Clarkson, E. N. K. 2003. A chitinozoan biostratigraphy of the Upper Ordovician and lower Silurian strata of the Girvan area, Midland Valley, Scotland. Transactions of the Royal Society of Edinburgh: Earth Sciences 93, 11134.

VANDENBRoucke, T. R. A., Williams, M., ZALASIEWICZ, J. A., DaVIES, J. R. \& WATERS, R. A. 2008. Integrated Upper Ordovician graptolite-chitinozoan biostratigraphy of the Cardigan and Whitland areas, southwest Wales. Geological Magazine 145, 199-214.

Webby, B. D., CoOper, R. A., BergströM, S. M. \& PAris, F. 2004. Chapter 2: Stratigraphic framework and time slices. In The Great Ordovician Biodiversification Event (eds B. D. Webby, M. L. Droser, F. Paris \& I. Percival), pp. 41-7. New York: Columbia University Press.

WhitTARD, W. F. 1931. The Geology of the Ordovician and Valentian rocks of the Shelve County, Shropshire. Proceedings of the Geologist's Association 42, 322-39.

WhitTARD, W. F. 1952. A geology of south Shropshire. Proceedings of the Geologist's Association 63, 143-97.

WHITTARD, W. F. 1979. An account of the Ordovician rocks of the Shelve Inlier in west Salop and part of north Powys. Bulletin of the British Museum Natural History (Geology) 33, 1-69.

Williams, A., Strachan, I., Bassett, D. A., Dean, W. T., Ingham, J. K., Wright, A. D. \& WhitTington, H. B. 1972. A Correlation of Ordovician rocks in the British Isles. Geological Society of London, Special Report No. 3 . 
Woodcock, N. H. 1984. The Pontesford Lineament, Welsh Borderland. Journal of the Geological Society, London 141, 1001-14.

Woodcock, N. H. \& GibBons, W. 1988. Is the Welsh Borderland Fault System a terrane boundary? Journal of the Geological Society of London 145, 915-23.

\section{Appendix. Sample localities}

\section{Shelve Inlier}

Shropshire, Welsh Borderland

The GPS measurements below are in the standard WGS84 reference system and the bedding is given as dip direction/dip readings.

\section{Samples from Lower Wood Brook, provided by Richard Bettley (see Bettley, unpub. Ph.D. thesis, Univ. Oxford, 1998)}

The geographical position of the samples along Lower Wood Brook is indicated on the map in Figure 2.

S 218/1: $17.39 \mathrm{~m}$ above the base of the section S 224/8: $17.71 \mathrm{~m}$ above the base of the section S 211/2 and S 211/4: $253.23 \mathrm{~m}$ above the base of the section

S 213/1 and S213/2: in between S211 and S214 (no accurate position provided)

S 214: $257.11 \mathrm{~m}$ above the base of the section S 217/1: $261.79 \mathrm{~m}$ above the base of the section S 228/1: $268.69 \mathrm{~m}$ above the base of the section S 227/2: $269.76 \mathrm{~m}$ above the base of the section S 230: $270.15 \mathrm{~m}$ above the base of the section S 231/6: $275.11 \mathrm{~m}$ above the base of the section $\mathrm{S} 232: 275.86 \mathrm{~m}$ above the base of the section S 244: c. $300 \mathrm{~m}$ above the base of the section

\section{Sample localities, Lower Wood Brook, 2002}

TVDB 02-102: Bettley's (unpub. Ph.D. thesis, Univ. Oxford, 1998) Locality S217 (Fig. 2), in a tributary to Lower Wood Brook, immediately south of the fence, $8.0 \mathrm{~m}$ upstream from the confluence with Lower Wood Brook; Rorrington Shale Formation

TVDB 02-101: Bettley's (unpub. Ph.D. thesis, Univ. Oxford, 1998) Locality S212 (Fig. 2), on the left bank of Lower Wood Brook, a little downstream of the fence on the left bank, in the middle part of the outcrop; Rorrington Shale Formation

TVDB 02-104: Bettley's (unpub. Ph.D. thesis, Univ. Oxford, 1998) Locality S217 (Fig. 2), in a tributary to Lower Wood Brook, immediately south of the fence, $2.7 \mathrm{~m}$ upstream from the confluence with Lower Wood Brook; Rorrington Shale Formation

TVDB 02-105: 9.5 paces downstream along Lower Wood Brook from the place where the fence described in TVDB 02-104 crosses Lower Wood Brook; Rorrington Shale Formation

TVDB 02-106: 9 paces downstream along Lower Wood Brook from the locality of TVDB $02-105$, in the middle of the river; Rorrington Shale Formation

TVDB 02-107: 9 paces downstream along Lower Wood Brook from Bettley's (unpub. Ph.D. thesis, Univ. Oxford, 1998) Locality S234 (Fig. 2) or 9 paces downstream along Lower Wood Brook from the place where the field boundary crosses Lower Wood Brook; Rorrington Shale Formation
TVDB 02-108: 35 paces downstream along Lower Wood Brook from the locality of TVDB 02-107; N $52^{\circ} 36.331^{\prime} \mathrm{W} 003^{\circ}$ 01.537'; Rorrington Shale Formation

TVDB 02-109: 59 paces downstream along Lower Wood Brook from the locality of TVDB 02-108; Rorrington Shale Formation

\section{Sample localities, Spy Wood Brook, 2002}

TVDB 02-159: Spy Wood Brook, right bank, 8 paces downstream of the confluence with the most northerly tributary shown in Figure 3; N 52 $33.541^{\prime} \mathrm{W} 003^{\circ}$ 03.371'; Rorrington Shale Formation

TVDB 02-162: 42 paces upstream in Dead Man's Dingle from TVDB 02-160; N 52 $33.411^{\prime} \mathrm{W} 003^{\circ} 03.471^{\prime}$; Rorrington Shale Formation

TVDB 02-160: Dead Man's Dingle, a tributary to Spy Wood Brook, 15 paces upstream from the confluence of the two aforementioned streams; N 52 $33.412^{\prime}$; W $003^{\circ} 03.510^{\prime}$; middle Rorrington Shale Formation; bedding $270 / 50$

TVDB 02-163: in the middle of Spy Wood Brook, $3 \mathrm{~m}$ downstream of a tributary, which is the first tributary south of Dead Man's Dingle; Rorrington Shale Formation; bedding 315/66; N 52॰ 33.329'; W $003^{\circ}$ $03.603^{\prime}$

TVDB 02-164: Spy Wood Brook, right bank, in the centre of the curve, where the stream takes a $c .90^{\circ}$ swing, top Rorrington Shale Formation

TVDB 02-165: Spy Wood Brook, 5 paces upstream from a $46 \mathrm{~cm}$ thick sandstone layer outcrop, in the transitional facies to the Spy Wood Sandstone Formation, stratigraphically $11 \mathrm{~cm}$ above the lowest clear $20 \mathrm{~cm}$ thick sandstone layer

TVDB 02-166: Spy Wood Brook, 14 paces downstream from the $46 \mathrm{~cm}$ thick sandstone layer outcrop described in TVDB 02-165; stratigraphically $11.40 \mathrm{~m}$ above the top of the same sandstone layer; Spy Wood Brook

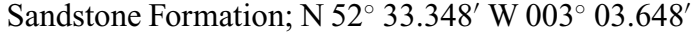

TVDB 02-167: Spy Wood Brook; top Spy Wood Brook Sandstone Formation, Bedding 160/40; N 52 33.358 ${ }^{\prime} \mathrm{W} 003^{\circ} 03.675^{\prime}$

TVDB 02-168: Spy Wood Brook; 10 to 20 m downstream from the TVDB 02-166 locality; transitional beds to the Aldress Shale Formation with obvious calcite veins; Bedding 278/85; N 52 $33.348^{\prime} \mathrm{W} 003^{\circ}$ $03.684^{\prime}$

TVDB 02-169: Spy Wood Brook, left bank, downstream from the TVDB 02-168 locality; N 52 $33.350^{\prime} \mathrm{W}$ $003^{\circ} 03.700^{\prime}$; Aldress Shale Formation

TVDB 02-170: Spy Wood Brook, left bank, downstream from the TVDB 02-169 locality; N 52 $33.360^{\prime} \mathrm{W}$ $003^{\circ}$ 03.716'; Aldress Shale Formation; Bedding $265 / 55$

TVDB 02-171: Spy Wood Brook, left bank, downstream from the TVDB 02-170 locality; N 52 $33.381^{\prime} \mathrm{W}$ $003^{\circ} 03.784^{\prime}$; Aldress Shale Formation

TVDB 02-172: Spy Wood Brook, left bank, downstream from the TVDB 02-171 locality; N $52^{\circ} 33.387^{\prime} \mathrm{W}$ $003^{\circ} 03.812^{\prime}$; Aldress Shale Formation

TVDB 02-173: Spy Wood Brook, left bank, downstream from the TVDB 02-172 locality; 22 paces upstream from the place where the brook disappears in concrete pipes below a forest track, or 29 paces upstream from the confluence with the Aldress Dingle; N $52^{\circ}$ 
33.403' W $003^{\circ} 03.894^{\prime}$; Aldress Shale Formation; Bedding 278/60

TVDB 02-174: Aldress Dingle, left bank, 8 paces downstream from a tributary to the Aldress Dingle (which is $c .100 \mathrm{~m}$ south of the Aldress Dingle-Spy wood Brook confluence); Aldress Shale Formation

\section{Onny Valley}

\section{Shropshire, Welsh Borderland}

List with all the labels used for the samples during the separate phases of the study, linked to the sample localities described in the literature by Jenkins (1967) and Turner (1982).

\begin{tabular}{|c|c|c|}
\hline Field label & UGent label & $\begin{array}{l}\text { Ancilletta label } \\
\text { unpub. (DEA thesis, } \\
\text { Univ. Liège, 1997) }\end{array}$ \\
\hline $91-15$ & 0001 / SV1 & S1 \\
\hline \multicolumn{3}{|c|}{$\begin{array}{l}\text { Turner (1982): OV/HS } / 2 ; 50 \mathrm{~m} \text { south of the middle of a foot } \\
\text { bridge; on the west side of the river floor; middle part of the } \\
\text { Harnage Shales Formation }\end{array}$} \\
\hline $90-08$ & JV 1 & $\mathrm{~S} 2$ \\
\hline \multicolumn{3}{|c|}{$\begin{array}{l}55 \mathrm{~m} \text { south of the middle of the bridge; middle part of the } \\
\text { Harnage Shale Formation }\end{array}$} \\
\hline $0-07$ & JV 3 & S3 \\
\hline \multirow{2}{*}{\multicolumn{3}{|c|}{$\begin{array}{l}\text { Jenkins (1967): C11; along the new road section; top part of the } \\
\text { Harnage Shale Formation - transitional facies to Horderley } \\
\text { Sandstone Formation }\end{array}$}} \\
\hline & & \\
\hline \multirow{2}{*}{\multicolumn{3}{|c|}{$\begin{array}{l}\text { along the new road section; basal part of the Horderley } \\
\text { Sandstone Formation }\end{array}$}} \\
\hline $\begin{array}{l}\text { Sandston } \\
0-09\end{array}$ & & \\
\hline \multicolumn{3}{|c|}{$\begin{array}{l}\text { Turner (1982): OV/LHS/1+2; } 55 \text { m north of bridge; } 20-30 \% \\
\text { up in the Horderley Formation }\end{array}$} \\
\hline $91-16$ & 0002 / SV2 & S6 \\
\hline \multicolumn{3}{|c|}{ Turner (1982): OV/LHS/1\&2 } \\
\hline \multicolumn{3}{|c|}{$\begin{array}{l}52 \mathrm{~m} \text { north of the stone bridge ('Glenn Burrell Bridge, } \\
\text { Longville') in a } 20 \mathrm{~cm} \text { thick shaly bed within the sandstones; } \\
\text { base of the Horderley Sandstone Formation }\end{array}$} \\
\hline $90-10$ & 0003 / SV3 & S7 \\
\hline \multicolumn{3}{|c|}{$\begin{array}{l}\text { Turner (1982): OV/UHS/1; Jenkins (1967) C } 10 ; 42 \mathrm{~m} \mathrm{~W} \text { of } \\
\text { barrier; } 13 \mathrm{~m} \text { west of New House (N-side of the river); high in } \\
\text { Horderley Sandstone Formation }\end{array}$} \\
\hline $0-11$ & 0004 / SV4 & S8 \\
\hline \multicolumn{3}{|c|}{$\begin{array}{l}15 \mathrm{~m} \text { east of the bridge; along the river; Alternata limestone } \\
\text { Formation }\end{array}$} \\
\hline
\end{tabular}

91-17

JV5

S9

$15 \mathrm{~m}$ east of the bridge; along the river; Alternata limestone Formation.

91-18

0005 / SV 5

S10

Jenkins (1967): C9; disused railway, $5 \mathrm{~m}$ south of a foot bridge,

$0.5 \mathrm{~m}$ above the ground level, Alternata Limestone Formation

91-19 0006 / SV6

S11

disused railway, $24 \mathrm{~m}$ south of a foot bridge, $1.5 \mathrm{~m}$ above the ground level, Alternata Limestone Formation

90-12 0007 / SV7

S12

Turner (1982): OV/LCL/1+2; road cut, W-side of a pond, bottom of the section, $60 \%$ high in the Cheney Longville Flags Formation

91-20

JV6

S13

Turner (1982): OV/LCL/1+2; Jenkins (1967): C8; W-side of a pond; road cut; lower half of the section; halfway in the Cheney Longville Flags Formation.

90-13 0008 / SV8

S14 Turner (1982): OV/LCL/1+2; road cut, W-side of pond; top part of the section, $9 \mathrm{~m}$ above sample $09-12 ; 60 \%$ high in the Cheney Longville Flags Formation

90-14 JV7

S15

Turner (1982): OV/AS/2, Jenkins (1697): C6; Onny River, low in the Acton Scott Formation.

91-21

JV 9

S16

Turner (1982): OV/0/2; Onny River cliff section; below water level and $4.2 \mathrm{~m}$ below the unconformity with the Silurian; Onny Formation

90-16

JV10

S17

Turner (1982): OV/O/2; Onny River cliff section; bottom of the outcrop; high in the Onny Formation

91-22 JV 8

S18

Turner (1982): OV/0/2; Onny River cliff section; 20 to $25 \mathrm{~cm}$ above sample $91-21$ and $4 \mathrm{~m}$ below the unconformity with the Silurian; Onny Formation.

90-17

0009 / SV9

Turner (1982): OV/0/1; Onny River cliff section; $1.5 \mathrm{~m}$ below unconformity with the Silurian and $2.5 \mathrm{~m}$ above sample $90-16$; high in Onny Formation

91-23 0010 / SV 10

$\mathrm{S} 20$ Turner (1982): OV/0/2; Onny River cliff section; $2.2 \mathrm{~m}$ above sample $91-22$ and $1.75 \mathrm{~m}$ below the unconformity with the Silurian; Onny Formation

TVDB 04-001: Onny River cliff section, easternmost side of the outcrop, $2 \mathrm{~m}$ west of the eastern edge of the cliff, $70 \mathrm{~cm}$ above the water level; stratigraphically 20 to $25 \mathrm{~cm}$ below the unconformity with the Silurian (sample with the trilobite Onnia ?superba); top Onny Formation

TVDB 04-004: Onny River; 27 paces east of the pedestrian bridge, which is immediately east of the former railway bridge across the Onny River; left bank; 4 paces east of the western edge of the outcrop; Harnage Shale Formation 